\title{
Propagation of Uncertainty in a Rotating Pipe Mechanism to Generate an Impinging Swirling Jet Flow for Heat Transfer from a Flat Plate
}

\author{
F.-J. Granados-Ortiz ${ }^{1}$, J. Ortega-Casanova ${ }^{2}$, and C.-H. Lai ${ }^{1}$ \\ ${ }^{1}$ School of Computing and Mathematical Sciences. University of Greenwich. Old Royal Naval College, \\ Park Row, London SE10 9LS, UK. \\ ${ }^{2}$ Fluid Mechanics Group. E.T.S. Ingeniería Industrial. Universidad de Málaga. C/ Dr. Ortiz Ramos \\ s/n. 29071 Málaga. Spain.
}

May 19, 2020

\begin{abstract}
In Computational Fluid Dynamics (CFD) studies composed of the coupling of different simulations, the uncertainty in one stage may be propagated to the following stage and affect the accuracy of the prediction. In this paper, a framework for uncertainty quantification in the computational heat transfer by forced convection is applied to the two-step simulation of the mechanical design of a swirling jet flow generated by a rotating pipe (Simulation 1) impinging on a flat plate (Simulation 2). This is the first probabilistic uncertainty analysis on computational heat transfer by impinging jets in the literature. The conclusion drawn from the analysis of this frequent engineering application is that the simulated system does not exhibit a significant sensitivity to stochastic variations of model input parameters, over the tested uncertainty ranges.

Additionally, a set of non-linear regression models for the stochastic velocity and turbulent profiles for the pipe nozzle are created and tested, since impinging jets for heat transfer at Reynolds number of $R e=23000$ are very frequent in the literature, but stochastic inlet conditions have never been provided. Numerical results demonstrate a negligible difference in the predicted convective heat transfer with respect to the use of the profiles simulated via CFD. These suggested surrogate models can be directly embedded onto other engineering applications (e.g. arrays of jets, jet flows impinging on plates with different shapes, inlet piping in combustion, chemical mixing, etc.) in which a realistic swirling flow under uncertainty can be of interest.
\end{abstract}

Keywords: Heat transfer; Impinging jets; CFD; Swirling jets; Uncertainty Quantification; Mathematical modelling

Journal: Engineering with Computers.

Corresponding author(s): J. Ortega-Casanova (jortega@uma.es). 


\section{Introduction and Motivation}

It is well known that Computational Fluid Dynamics (CFD) is a powerful tool in fluid-related fields such as optimisation [1, 2], aerospace \& aerodynamics industry [3, 4, 5], fire safety modelling [6], heat transfer [7] or nuclear energy [8], amongst many others. Much effort has been spent to develop numerical algorithms for CFD, leading to more reliable simulations for decision-making and validation purposes, where uncertainty plays an important role.

In experimental work, uncertainty and error measurements are often given but, when performing CFD simulations, this is not a regular practice. If one needs to provide reliable results, this should be a must to offer the most complete overview by including confidence measures. Generally speaking, in CFD simulations, boundary conditions and geometries are often imposed, without considering the effect on performance that real-life stochastic variations in geometry and boundary conditions may have. Therefore, an option to take into account the stochasticity of some parameters is to use a stochastic analysis instead of a deterministic approach. For this purpose, uncertain inputs are mathematically modelled by using probabilistic distributions derived from experimental data. This is sometimes unavailable and scientists may model the relevant input uncertainties by means of intervals, as well as the study of this propagation, mostly based on experience.

The work presented in this paper is based on the deterministic CFD simulation validated in [9], whose turbulence model and mesh discretisation errors (Grid Convergence Index, GCI [10]) were also discussed and validated in several computational works of heat transfer by swirling impinging jets by the authors $[7,11,12]$. For an efficient computation, the CFD simulation was carried out in two stages: Simulation 1 \& 2 (see Figure 1). Simulation 1 generates the swirling flow to be used as inlet condition in Simulation 2, where the heat transfer from the flat plate to the swirling jet is computed. This two-step approach is an efficient alternative to work with different turbulent models (the flow regime in the pipe is different to the flow regime over the plate) as well as to impose periodic boundary conditions onto the pipe to get a fully-developed flow (this avoids a large computational domain) [9]. To properly solve both problems, different turbulent models are tested. The turbulence models with the best performance were the Reynolds Stress Models (RSM) for Simulation 1, and the Shear Stress Transport (SST) $k-\omega$ for Simulation 2. More details are given in Section 3, by means of a brief description of the set-up, but the reader is referred to $[9,7,11,12]$ for further information about the successful application of these turbulent models in swirling jet flows.

As aforementioned, the main interest is to simulate a swirling flow to enhance the heat transfer on the flat plate. It has been shown in $[7,13,12]$ that the addition of swirl to impinging jets can increase heat transfer. Note that the swirl can be generated in different ways, such as by using spiral ducts [14, 15, 13], angled blades at the tip [16], agitation by stirrer blades, or by a rotating pipe [17]. Depending on the generating mechanism, the outflow will have different patterns and, thereby, the jet spread rate will be different. This feature is very influential and the degree of swirl has a dramatic effect on the heat transfer by modifying the shear layer growth and instabilities, entrainment of ambient air and other properties [15].

As discussed in [9], there is a large amount of research on non-swirling impinging jets [18, 19, $20,21,22]$, and it has been also shown in several works in the literature $[13,15,14,12,7,23]$ that the addition of swirl to these impinging jets can increase the heat transfer under certain conditions. 
Additionally, the existence of some particularities on this type of heat transfer problem also led to important recent investigations on the most appropriate modelling. For instance, only few investigations address the use of Large Eddy Simulations (LES) in heat transfer by impingement [24, 25, 26]. Despite the classic good performance of this high-fidelity simulation for the flow structures in jets, in the present problem set-up LES usually do not match very accurately the experimental data for the radial Nusselt distribution on the plate [27, 28]. Actually, for short nozzle-to-plate distances, the difficulty to match LES data and explain the secondary peak in the radial Nusselt number is an important investigation attempted by several authors [25, 26, 27].

Recent work such as [29] developed a new version of the SST $k-\omega$ and applied it to impinging slot jets for heat transfer by considering the cross-diffusion term and Kato-Launder model. They found that the model has increased sensitivity to pressure gradients, of high relevance in heat transfer by impingement. Additionally, in [30] a similar turbulence model is developed by a combination of the standard SST turbulence model with the crossflow transition model, intermittency transition model and the Kato-Launder model. Their results have a good match with experimental and numerical high-fidelity data, concluding that the customised turbulence model accurately simulate the heat transfer and flow structures of the impinging slot jet problem. In [31], the effect of heat transfer from a flat plate to a swirling jet is analysed according to the Burger's vortex, finding optimal configurations considering different vortex parameters (swirl intensity and vortex cores). Other interesting investigations are [32, 33], where the heat transfer of an impinging swirling jet at high Reynolds $(R e=35000)$ is studied both numerically and experimentally, and their results were used for CFD validation in the predecessor of the present work, [9]. As pointed out in [26], although the computational study of the heat transfer by impinging jets has been an extensive area of research for decades, this type of problem is still a challenging investigation that enhances the importance of the present study.

For the purpose of simulation, a probabilistic approach to estimate uncertainty provides a more complete overview on the reliability of the numerical computation than a deterministic single-point simulation. This is because the stochastic variance of some parameters is taken into account in the simulation and may have effect on the performance. To our knowledge, there is no previous literature on the effect of simulating impinging jet flows for heat transfer under uncertain conditions, apart from the early stage work presented by the authors in a conference [34]. This is a motivation to provide a framework for these complex problems, since the quantification of uncertainties should be an important common practice in computational engineering.

Regarding the sources of uncertainty, these are classified as aleatoric or epistemic. Aleatoric uncertainty is considered as implicit to the natural stochasticity in a physical system or quantity. It is also referred to in the literature as irreducible uncertainty, inherent uncertainty, variability and stochastic uncertainty [35]. On the other hand, epistemic uncertainty is product of the imprecision in the modelling as result of a lack of knowledge. This type of uncertainty could, in theory, be reduced if additional information can be added [36]. Epistemic uncertainty associated to the fidelity of the simulation can have a significant effect, and different sources of uncertainty may be actually related. For instance, in [37] uncertainty is quantified by using both Reynolds Averaged Navier Stokes (RANS) and LES in a heat transfer problem demonstrating that there is an important link between aleatoric and epistemic uncertainties. 


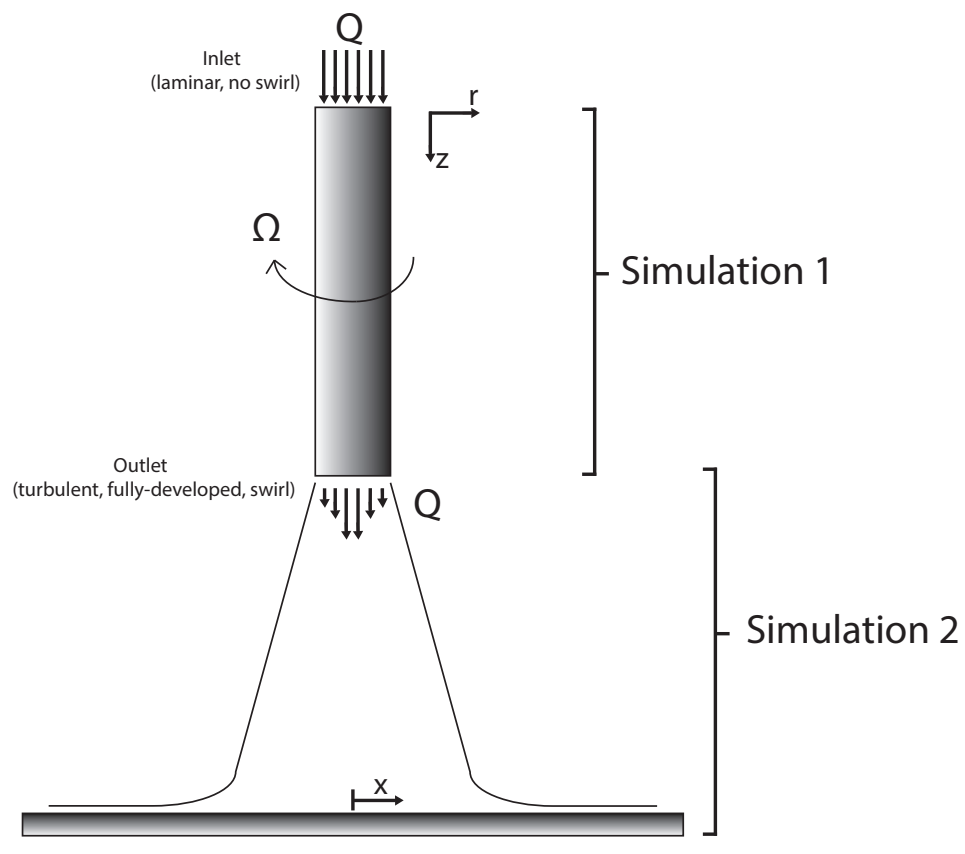

Figure 1: Sketch of Simulation 182 . Note that the $x$ and $r$ axis are the same in practice and have their respective origins in the axisymmetry axis, but for the sake of avoiding confusion in the following plots, $r$ is used in Simulation 1 and $x$ for Simulation 2.

Although the application of Uncertainty Quantification (UQ) to fluid dynamics and heat transfer is increasing in popularity exponentially [38, 39, 40, 41, 42], there are just few documented applications on applying uncertainty quantification to computational simulations of swirling flows $[43,44]$. In [43] uncertainty in thermoacoustic instabilities in a swirled stabilized combustor were studied. The motivation of analysing such stochasticity is that the impact of uncertainty could be noticed in the stability modes. This exhibits the importance of a stochastic modelling approach to measure the probability of a mode to be unstable with respect to the input random variables. This is of high relevance in combustor science, since extreme combustion instabilities can highly damage the system, as mentioned in their work. In [44], a swirling flow with swirl intensity, $S$, ranging from 0 to 0.6 confined in a pipe is simulated by means of both RANS and LES. The pipe is rotating with a Reynolds number of $R e=30000$, and it undergoes a sudden expansion. The numerical quantification of uncertainty was found to be very close to the reported experimental one, by means of both RANS with the $k-\epsilon$ model and LES with the Smagorinsky model. However, due to the lower fidelity of RANS simulations, these provided the least accurate and most sensitive results in the simulations. This work is very close to our problem under study (containing RANS simulations, rotating pipe, swirling flow suddenly expanded, similar $R e$, similar $S$ values). Their outcomes encouraged us to undertake the quantification of experimental uncertainties in our simulations. The impact of uncertainty in CFD simulations of jets has been also studied by the authors in [5], where the simulation of a compressible jet flow under uncertain conditions is analysed, demonstrating that there is a relationship between the input random variables and the spatial distribution of pressure and velocity arising due to the propagation of uncertainty in the simulation. Other papers that also offered a reference and motivation are [45], where synthetic 
jets by means of polynomial chaos are studied, [46] where underexpanded jets in a crossflow for turbulent mixing are investigated, and [47], where uncertainty estimation is developed in RANS simulations of high-speed aircraft nozzle jets. In several papers $[46,47,48]$, a methodology to deal with the well-known epistemic uncertainty in turbulence models is outlined and tested, by means of eigenvalue and eigenvector perturbations. In their work, the perturbation of the eigenvalues of the Reynolds stress anisotropy tensor is modelled by the position in a barycentric triangle map, whose corners stand for the limiting states of turbulence anisotropy. The eigenvector perturbation is made to change the Reynolds stress tensor alignment to find the extremal alignments with the mean strain. Throughout these papers it is highlighted the importance of providing uncertainty bounds in RANS simulations, and the results suggest that their uncertainty estimation method can account most of the model inadequacy. During the literature survey no applications of UQ to heat transfer by impinging jet flows were found.

The structure of this paper is as follows. In Sections 2 and 3, the methodology and a description of the problem are given as an overview. In Section 4, the uncertainty quantification process is described, in order to understand Stochastic Collocation Method and the use of sparse grids. The considered sources of uncertainty are also described in this section. In Section 5, the coupling process between Simulation 1 and Simulation 2 to simulate the entire domain is explained. In Section 6, the steps followed to model the outflow profiles are discussed and the definitive suggested regression models are given. After this modelling process, in Section 7 a comparison is made between the propagated uncertainties with and without implementing the models for the pipe outflow profiles. In this section, different probabilistic distributions for the inputs on the surrogate models are also tested, for the UQ purposes of this work. Finally, in Section 8, the relevant conclusions of this work are given. In Appendix A, polynomial models for the coefficients of the non-linear regression models are shown, and in Appendix B a piece of code is provided for the implementation of the models by a User Defined Function (UDF) in FLUENT.

\section{Methodology}

The aim of the Uncertainty Quantification is to provide confidence measures on how the output of a model, say $\hat{y}$, is varied due to the variability of its inputs, say $\hat{\xi}_{i}$ [49] (see Fig. 2). In the present work, our main investigation is restricted to aleatoric uncertainties arising due to experimental errors or variability. For this reason, most experimentalists provide error bars as reliability measures. However, output CFD data usually lacks reliable confidence indicators, which can be obtained by including experimental uncertainty (aleatoric uncertainty) in input CFD data as we do in this investigation. Any epistemic uncertainty from the use and calibration of turbulence models is not considered. However, this study contains modelling work, which consists of the search of mathematical models for the inlet profiles for Simulation 2. Since the considered aleatoric uncertainties are actually the same with and without the models, and the UQ method is also the same, the impact of the epistemic uncertainty associated to the non-linear regression models is being quantified. Due to the fact that the concept of epistemic uncertainty may evoke to several sources to the reader, this specific inaccuracy is referred to as modelling uncertainty in this manuscript. 
Once the input uncertainties are modelled, it is necessary to find an appropriate UQ method. These methods can be either intrusive or non-intrusive. A non-intrusive approach is chosen since this does not require additional code implementation in the solver and can deal with any model as a black-box (FLUENT software in our case). On that basis, Monte-Carlo simulations [50] are a reliable possibility. This non-intrusive method is based on a random sampling on the input uncertainties in order to obtain enough outputs to build the statistical output data. As the convergence of the method is slow, being of order $O\left(1 / \sqrt{N_{s}}\right)$, with $N_{s}$ the number of samples, in order to reduce $N_{s}$ and increase the efficiency of the method, other sampling methods are available in literature such as the Latin Hypercube [51], the quasi-random Halton [52] or the Sobol sequences [53], amongst others. These sampling techniques optimise the sampling by taking also into account previous positions of the samples in the stochastic space, and the error is essentially of order $O\left(1 / N_{s}\right)$. It is important to point out that this efficiency is mostly noticed for moderate dimensions of the stochastic space, as for high dimensional problems they behave similarly to Monte-Carlo (which is dimension independent).

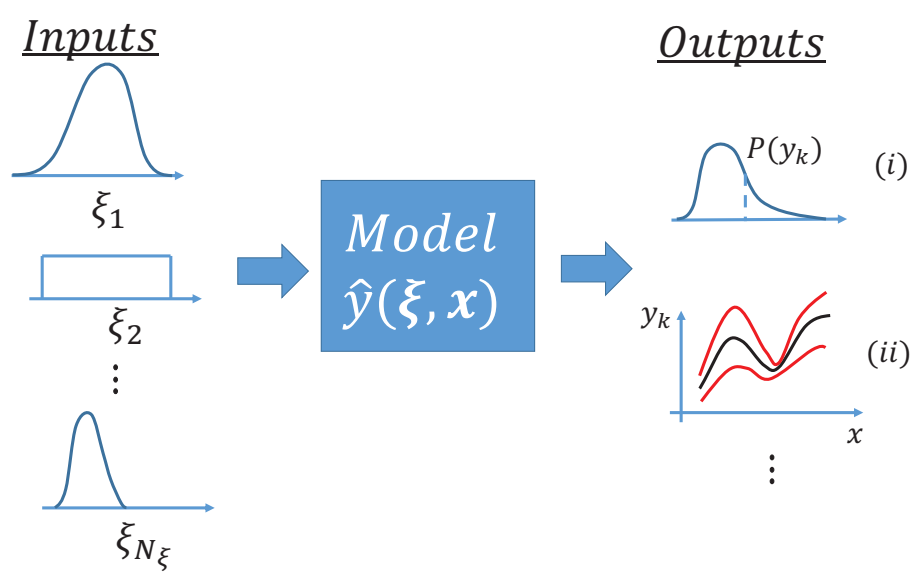

Figure 2: Propagation of uncertainties in a mathematical model.

Sampling based methods are, therefore, a good choice for UQ. However, these usually require a large number of model evaluations. This is the main disadvantage when performing UQ on $\mathrm{CFD}$, as each model evaluation requires a large computational time, being often unaffordable. To overcome this drawback, the Stochastic Collocation Method (SCM) [54] is implemented. This calculates the statistical moments of the output by dedicated quadrature techniques on tensor grids. SCM has better performance than sampling methods especially for low dimensions in the stochastic parameter space. When the dimension is high, the cost leads to the so-called Curse of Dimensionality, as the number of collocation points to evaluate increases exponentially, and it is preferable to consider other methods. An efficient way to mitigate the needs of using many collocation points is to use sparse grids [55], for which the accuracy (that is to say the number of collocation points) can be consistently increased until convergence is achieved.

SCM based approaches have been used in several applications in the literature. This nonintrusive method has been successfully applied to problems such as elliptic partial differential equations with random input data [56], supersonic aircraft jets [4] or cardiovascular research [57]. 
In some UQ methods, including SCM, the exact response can be approximated by creating a surrogate model. Then, sampling techniques can be applied on the surrogate to recover more statistical information, such as probabilistic distributions of the outputs. For this reason, two types of input probabilistic distributions were tested in the present work to generate the random inputs on the surrogates: a uniform and a normal distribution. As experimental uncertainty data is not available to build the empirical probabilistic functions, to try two different distributions provide some information about the impact of non-linearity and high-order effects in the propagation of uncertainty through the CFD simulation. The random variables are sampled to evaluate the SCM response surface and get converged probabilistic distributions of the outputs.

Since the swirling flow generation in Simulation 1 is decoupled from Simulation 2, it is interesting for reliability reasons to find a way to characterise the outflow velocity and turbulence profiles from Simulation 1 under uncertain conditions, to impose that data onto Simulation 2. This is the objective in Section 7.3, where non-linear regression models are sought. A numerical computation of the uncertainty of the outlet profiles from Simulation 1 is done by means of SCM in the present paper, at different values of the normalised radial coordinate $r / R$, where $r$ is the radial coordinate and $R$ is the radius of the pipe. However, to quantify the uncertainty in the output of Simulation 2, several profiles should be systematically generated from Simulation 1 and then input as boundary condition to Simulation 2. The alternative to avoid Simulation 1 and provide functions to generate the profiles as input in Simulation 2 is investigated in this work. These functions can be coded into FLUENT by means of a User Defined Function (UDF) to any CFD problem, in contrast to coding a SCM surrogate for every $r / R$ location, which is a very cumbersome option, as well as potential source of human errors.

Impinging jets at Reynolds number of $R e=23000$ have been one of the most studied in the literature for years on end, e.g. in $[14,58,59,31,26,60,18,27,9,13,25]$. Hence, it is useful for future research to have stochastic/non-stochastic profiles ready to input as boundary condition to CFD simulations. The use of algebraic functions for modelling jet flow profiles is an established practice $[61,62,63,64]$, for instance to perform stability analysis of the jet plume [65, 66]; or in topics closer to the present paper as the jet in [16], whose swirling jet empirical functions were successfully used as inlet profiles to simulations in $[7,11,12]$.

\section{A brief description of the set-up simulated by CFD}

In this paper, few details on the CFD configuration are given, since a detailed numerical investigation and validation of this mechanical system was developed in [9]. In this study, the impinging swirling jet is created by using a rotating pipe with a fully-developed flow at its exit. The inlet is a uniform flow with Reynolds number $R e=\frac{4 \rho Q}{\pi D \mu}=23000$, where $\rho$ is the fluid density, $Q$ is the volume-flow rate, $D$ is the pipe diameter and $\mu$ is the dynamic viscosity; whilst the outflow is a fully developed turbulent flow. This flow spreads from the exit of the pipe (nozzle) and impinges on the heated flat plate below, located orthogonally at a dimensionless distance $H / D=5$, where $H$ is the distance between the nozzle and the plate. The Prandtl number is $\operatorname{Pr}=\frac{\nu}{\alpha}=0.71$, where 
$\nu$ is the kinematic viscosity of the fluid and $\alpha$ is the thermal diffusivity. The Swirl number is set to $S=\frac{\pi D^{3} \Omega}{8 Q}=1$, with $\Omega$ the angular velocity of the pipe. Since dimensionless numbers are used to describe the working conditions, the angular velocity can be chosen at any value as long as the $S=1, R e=23000$ and $\operatorname{Pr}=0.71$ conditions are kept. For instance, air can be chosen as working fluid (air has $\operatorname{Pr}=0.71$ ), so $\rho$ and $\mu$ are those values corresponding to air. Then, a pipe of arbitrary diameter $D$ needs a fixed volume-flow rate $Q$ to achieve $\operatorname{Re}=23000$. Once $Q$ is known, $\Omega$ can be solved from the Swirl number definition by taking into account that $S=1$.

Prior to the simulation of the heat transfer, the velocity and turbulent profiles of the impinging swirling jet are to be produced in a separate simulation of a rotating pipe. This is Simulation 1, shown in Fig. 1. In Fig. 3(a), the pipe problem is depicted, for which a 2D RANS simulation was developed in FLUENT. The flow under study is axisymmetric, steady, incompressible and becomes fully-developed. To obtain the fully-developed pipe flow from a uniform inflow requires a pipe length greater than a specific characteristic one. In order to reduce the cost of this computation, a piece of pipe has been simulated with periodic boundary conditions, with a mass-flow rate corresponding to $R e=23000$ and $S=1$. The turbulence model used in this simulation was the Reynolds Stress Model (RSM). The finite-volume discretisation used the axisymmetric mesh of the pipe shown in Fig. 3(b). The mesh size is $\left[n_{r} \times n_{z}\right]=68 \times 450$ cells, having a dimensionless wall distance of $y^{+}<1$ along the wall of the pipe. The mesh convergence of Simulation 1 was reported in [9], where three grids of $\left[n_{r} \times n_{z}\right]=30 \times 200,45 \times 300$ and $68 \times 450$ (mesh in the current work) were analysed. The grid discretisation error for the $68 \times 450$ mesh was reported as a $0.2 \%$, by means of the Grid Convergence Index (GCI) method. Regarding the quality of the simulation, one can find in the literature that other authors generated similarly the rotating pipe profiles that we obtained in our deterministic CFD simulation. These authors also used the RSM model for the rotating pipe flow simulation $[67,68,69]$, as this model exhibits the best performance. In addition, the experimental results reported by $[17,70]$ were used to validate the accuracy of the CFD simulation in [9], being very interesting to observe a nearly perfect match for the axial velocity profile. Simulation 1 was found to be very accurate even at very high Reynolds number $(R e=50000)$, validated with experimental data from [70], as shown in the validation plots in Fig. 4.

Once Simulation 1 is completed, its output is used as inlet boundary condition for Simulation 2. In Fig. 5, the detail of its discretisation and boundary conditions of the $2 \mathrm{D}$ axysimmetric simulation are depicted. The computational mesh is in this case $\left[n_{x} \times n_{h}\right]=140 \times 250$ cells, ensuring an $y^{+}<1$ all along the plate. The mesh convergence analysis of this simulation was reported in [9], where the same mesh was used, being the reported discretisation error (calculated with the GCI method) of Simulation 2 equals to a $0.4 \%$. The dimensionless nozzle-to-plate distance is $H / D=5$ and a piece of the swirling pipe of length $D$ has been included in the domain in order to enable the pressure field to properly develop inside the pipe prior the expansion Simulation 1. For the sake of clarity, the $x$ axis is the same as the $r$ one, but different notation is used to avoid confusion between the parameters at the exit of the pipe and those on the flat plate. In Fig. 5 since the flow is not confined, the upper boundary is not a solid wall but a pressure boundary where the usual boundary condition of known constant pressure is imposed, whereas null Neumann boundary 


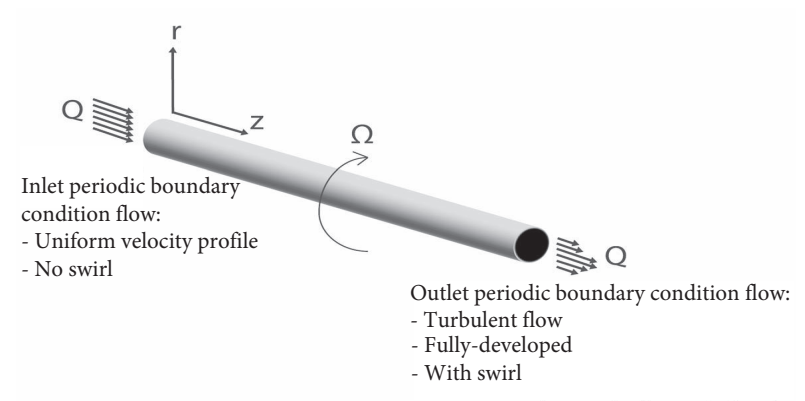

(a)

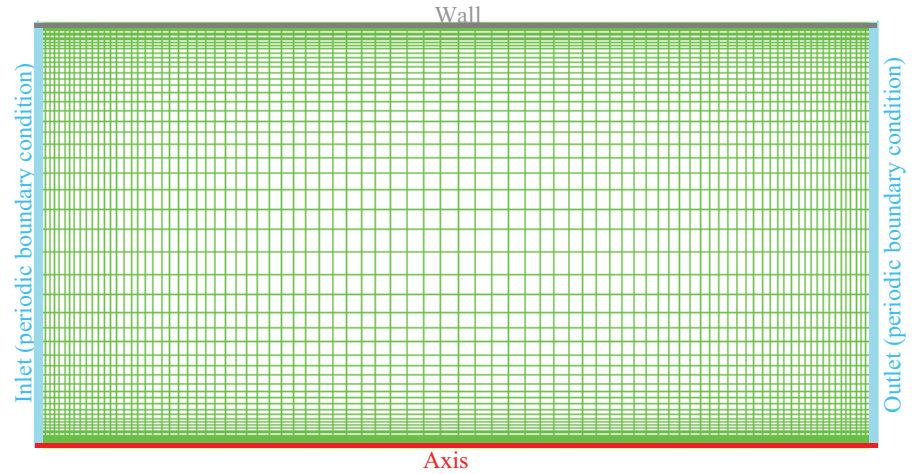

(b)

Figure 3: (a) Sketch of the swirling flow generator by a rotating pipe. The axial periodic flow is initialised with a uniform profile and without swirl, and develops over time as turbulent fullydeveloped pipe flow with swirl. (b) Axisymmetric 2D mesh of the rotating pipe. In the simulation, the flow goes from left (inlet boundary condition) to right (outlet boundary condition) to impose the periodic condition. The bottom side is the axis (axis boundary condition) and the top side is the pipe wall (wall boundary condition with rotation imposed).

conditions are used for the remaining fluid magnitudes, also the turbulent ones, which means that these are extrapolated from interior cells to satisfy that their first derivative is zero. Regarding the turbulence model, the SST $k-\omega$ has been used. Simulation 2 has been tested and validated in [9] against experimental and numerical data at $R e=23000$, but also at a higher Reynolds number of $R e=35000$ with several swirl intensities, using experimental data from [33], as shown in Fig. 6 , both for the most extreme case of $H / D=2$. To sum up, both the turbulent models RSM for the rotating pipe and the SST $k-\omega$ for the impinging problem have been exhaustively analysed and validated in [9]. Additionally, the mesh convergence and turbulent models of the impinging jet simulation were validated against experimental data in $[9,7,11,12]$. The authors suggest to see these three last publications for further information on the computational features in these simulations, including the grid convergence analysis, comparisons against experimental results and other tested turbulence models.

Once the deterministic CFD simulations are ready, these can be run a number of times with different values of the input parameters to perform the uncertainty analysis. In this work, the chosen method is the Stochastic Collocation, which is formally described next.

\section{Uncertainty Quantification}

\subsection{Stochastic Collocation Method}

The method implemented here for UQ is the Non-Intrusive Stochastic Collocation Method (SCM). It was originally developed by Mathelin and Hussaini [54] at NASA as alternative to other UQ methods, which demanded higher costs. SCM represents a very efficient option for lower dimension problems in comparison with sampling techniques such as e.g. Monte-Carlo. For higher dimension 

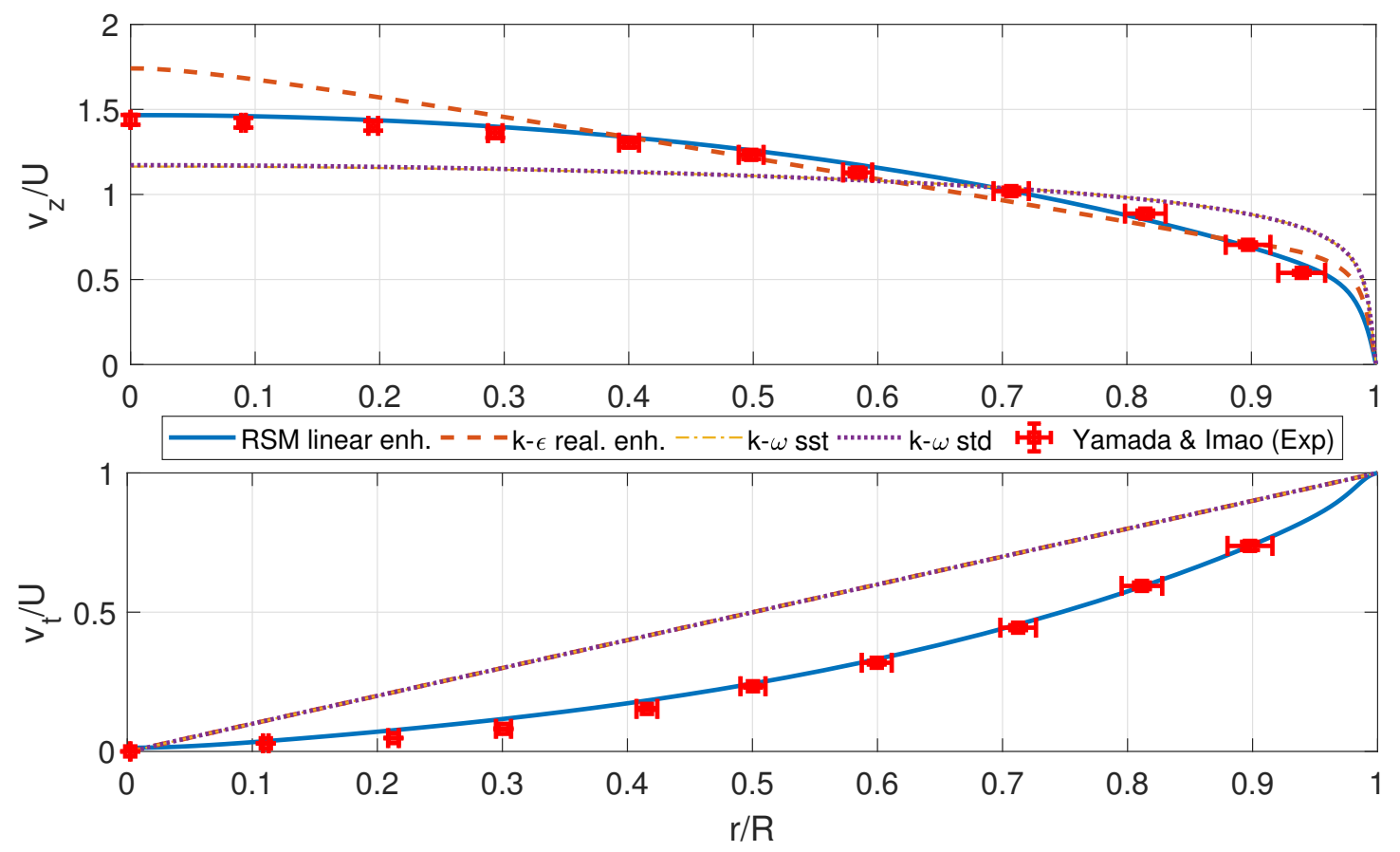

Figure 4: Validation of axial and azimuthal velocity profiles of the fully-developed swirling flow with $R e=50000$ and $S=1$. The experimental data Yamada \& Imao (Exp) are taken from [70] with $2 \%$ error bars. RSM linear enh stands for RSM with Linear Pressure-Strain and Enhanced Wall Treatment, $k-\epsilon$ real. enh. stands for the $k-\epsilon$ realizable turbulence model with Enhanced Wall Treatment, $k-\omega$ sst and $k-\omega$ std are the $k-\omega$ turbulence models with option Shear Stress Transport and Standard, respectively. All simulations were developed in [9].

problems, sampling techniques tend to be more suitable.

Consider the differential operator on an output of interest of a stationary problem, $y(\mathbf{x}, \boldsymbol{\xi}(\eta))$ as

$$
\mathcal{L}(\mathbf{x}, \boldsymbol{\xi}(\eta) ; y(\mathbf{x}, \boldsymbol{\xi}(\eta)))=\mathcal{Q}(\mathbf{x}, \boldsymbol{\xi}(\eta))
$$

with $\mathcal{L}$ and $\mathcal{Q}$ differential operators on $\mathcal{D} \times \Xi$, where $\mathbf{x} \in \mathcal{D} \subset \mathbb{R}^{d}, d \in\{1,2,3\}$. $\eta$ denotes events in the complete probabilistic space $(\hat{\Omega}, \hat{\mathcal{F}}, \hat{P})$, with $\hat{\mathcal{F}} \subset 2^{\hat{\Omega}}$ the $\sigma$-algebra of subsets of $\hat{\Omega}$ and $\hat{P}$ a probability measure. $\Xi \subset \mathbb{R}^{N_{\xi}}$, is the stochastic space on which the random variables $\boldsymbol{\xi}(\eta)$ are defined and $N_{\xi}$ stands for the number of random variables (two in our case under study). The objective is to find the $i$-th statistical moments, $\mu(\mathbf{x}, \boldsymbol{\xi})_{i}^{y}$, of the output of the model by

$$
\mu(\mathbf{x}, \boldsymbol{\xi})_{i}^{y}=\int_{\Xi} y(\mathbf{x}, \boldsymbol{\xi})^{i} f_{\xi}(\boldsymbol{\xi}) d \boldsymbol{\xi}
$$

with $f_{\xi}(\boldsymbol{\xi})$ standing for the density function $f_{\xi}: \xi \mapsto \mathbb{R}_{+}$. In many applications this is not sufficient and incomplete information of the variable of interest is given. The reason is that two very different probabilistic distributions can have, for instance, the same mean and standard 


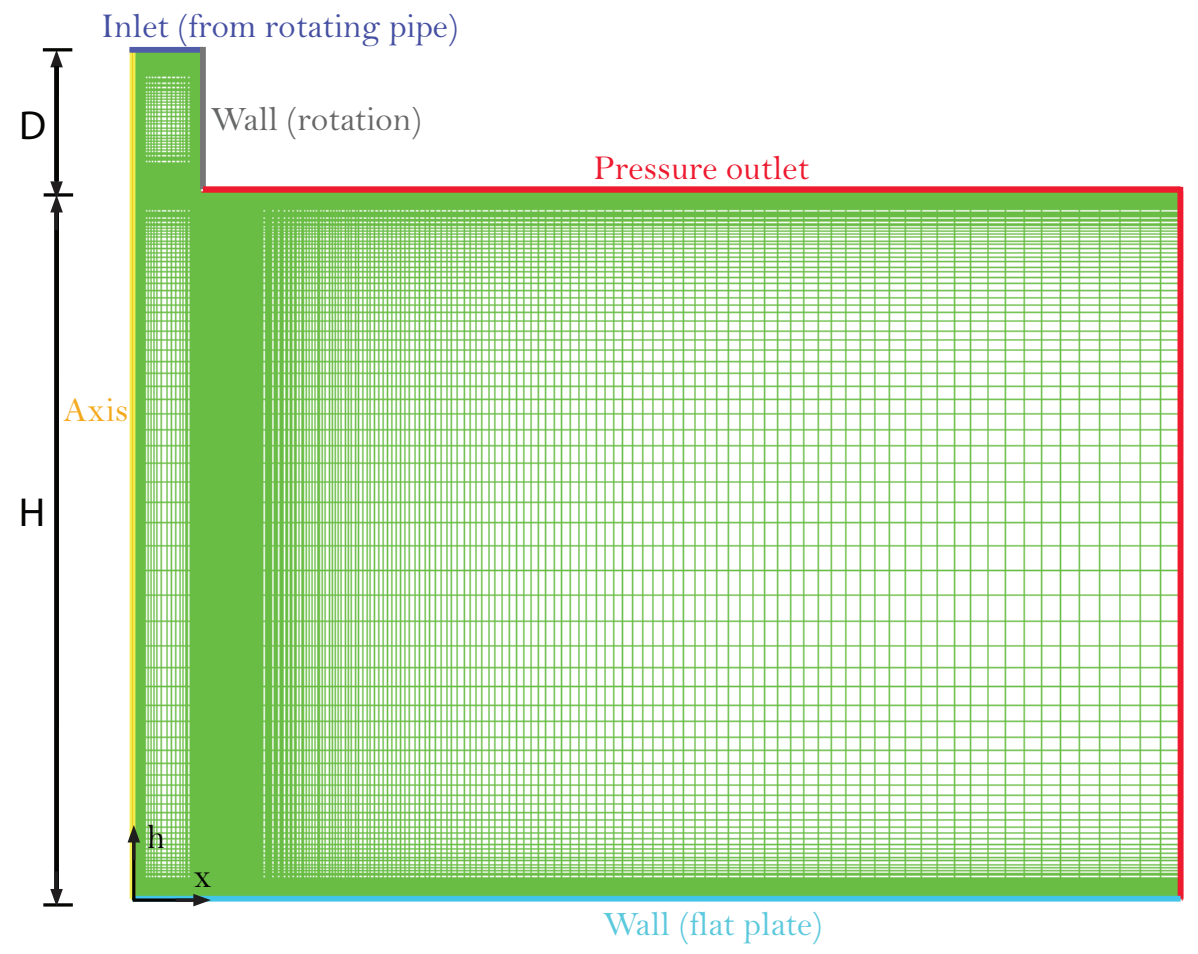

Figure 5: Sketch of the impinging jet flow and the boundary conditions chosen in FLUENT. A portion of the pipe of length $D$ is simulated for a less abrupt adaptation of the inlet from Simulation 1 in Simulation 2.

deviation. Therefore, this can be misleading when further information is required, such as finding out whether the solution is multimodal. Consequently, the probabilistic distribution of $y(\mathbf{x}, \boldsymbol{\xi})$ is often sought by sampling surrogates as in this manuscript.

When implementing SCM, special attention must be paid to the probabilistic density function of the random variables $\xi \in \Xi$, as we have to perform a mathematical transformation from the physical random variable space to an artificial stochastic space, known as $\alpha$-domain or $\alpha$-space:

$$
\alpha=\mathcal{S}(\xi)
$$

This transformation is a difference with respect to other UQ methods, as the stochastic space $\alpha$ is defined in the domain of Lagrange interpolating polynomials in $[-1,1] .{ }^{1}$ It is hence useful that, for each collocation point, the CFD problem is solved deterministically and the solution can be reconstructed by

$$
\hat{y}(\mathbf{x}, \alpha) \simeq \sum_{j=1}^{N_{q}} y_{j}(\mathbf{x}) l_{j}\left(\mathcal{S}^{-1}(\boldsymbol{\alpha})\right)=\sum_{j=1}^{N_{q}} y_{j}(\mathbf{x}) l_{j}(\alpha),
$$

where $y_{j}(\mathbf{x})$ are the deterministic solutions from $y\left(\mathbf{x}, \xi_{j}\right)$ now transformed into $y\left(\mathbf{x}, \alpha_{j}\right), N_{q}$ the number of collocation points and $l_{j}$ the Lagrange interpolation polynomials defined in the new

\footnotetext{
${ }^{1}$ It is interesting to point out that in several papers [54, 71], $\alpha$ is referred to as an artificial stochastic space, but the space is in fact $\Gamma$, as $\alpha \in \Gamma$. In this work, $\Gamma$ will be referred to as the $\alpha$-space in order to be consistent with the existing notation in the literature.
} 


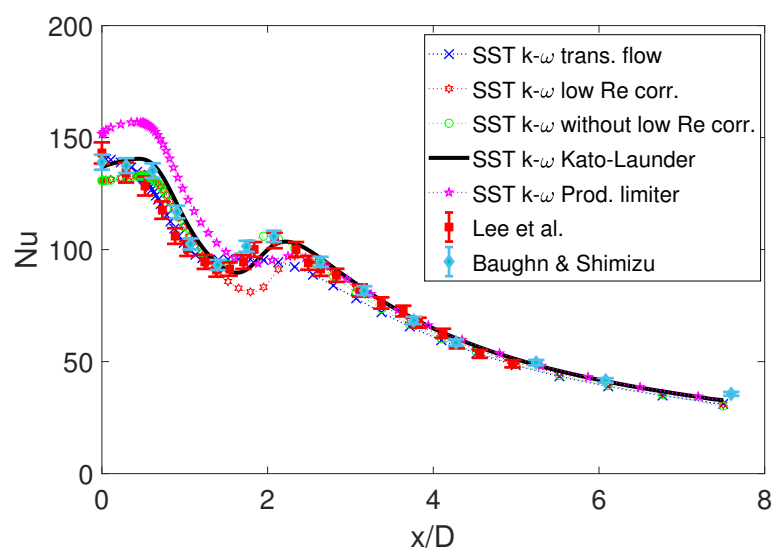

(a)

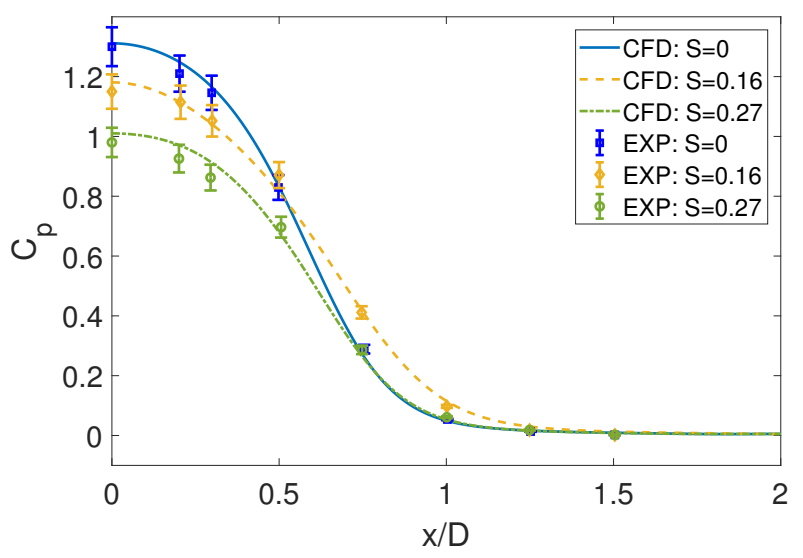

(b)

Figure 6: (a) Validation of the turbulence model for $H / D=2$ and $R e=23000$. The different configurations of the SST $k-\omega$ turbulence model correspond to: with transitional flow, with Low Reynolds correction, without Low Reynolds correction, Transition SST with the Production Kato-Launder option and Transition SST with the Production Limiter option. Lee et al. [14] experimental data have their reported 3.3\% uncertainty; and Baughn 6 Shimizu [18] includes their $2.4 \%$ of uncertainty. (b) Validation of CFD simulation by pressure coefficient at $R e=35000$ and $S=0,0.16$ and 0.27. The experimental data (EXP) are taken from [33], including their 5\% uncertainty in the error bars. All simulations were developed in [9].

stochastic space. Note that $\mathcal{S}^{-1}(\boldsymbol{\alpha})$ denotes the inverse mathematical transformation to $\alpha \in \Gamma$. The integral of Eq. (2) now can be approximated as

$$
\mu(\mathbf{x}, \boldsymbol{\alpha})_{i}^{\hat{y}}=\sum_{j=1}^{N_{q}} y_{j}(\mathbf{x})^{i} \int_{\Gamma} l_{j}(\boldsymbol{\alpha}) f_{\xi}(\boldsymbol{\alpha}) J(\boldsymbol{\alpha}) d \boldsymbol{\alpha},
$$

where $J(\boldsymbol{\alpha})$ the Jacobian of the differential transformation. Finally, it can be numerically computed by quadrature as

$$
\mu(\mathbf{x}, \boldsymbol{\alpha})_{i}^{\hat{y}} \simeq \sum_{j=1}^{N_{q}} y_{j}(\mathbf{x})^{i} \sum_{k=1}^{N_{q}} l_{j}\left(\alpha_{k}\right) f_{\xi}\left(\alpha_{k}\right) J\left(\alpha_{k}\right) z_{k},
$$

with $z_{k}$ and $\alpha_{k}$ standing for the quadrature weights and points respectively. Both the quadrature and collocation points are the same in this work. For the Lagrange interpolating polynomials, must be taken into account that $l_{i}\left(\alpha_{j}\right)=\delta_{i j}$ for $i, j=\left[1,2, \ldots, N_{q}\right]$. Therefore, with these assumptions, the integral from Eq. (6) above can be finally rewritten for a uniform probabilistic input defined in $[-1,1]$ as

$$
\mu(\mathbf{x}, \boldsymbol{\alpha})_{i}^{\hat{y}} \simeq \frac{1}{2} \sum_{j=1}^{N_{q}} y_{j}(\mathbf{x})^{i} z_{j} .
$$

The choice of collocation points is an important matter. If tensor grids are chosen, the computational cost would be very high, especially when $N_{\xi}$ is not low. A very efficient alternative 
is the use of sparse grids. In the present paper, the collocation points of the sparse grids have been determined according to the Clenshaw-Curtis (C-C) quadrature nested rule [72] and Smolyak construction [73]. With sparse grids, several levels of accuracy were tried in the problem, in order to test the convergence. In Fig. 7 the evolution of the sparse grid collocation points is shown up to the fourth level for $N_{\xi}=2$.
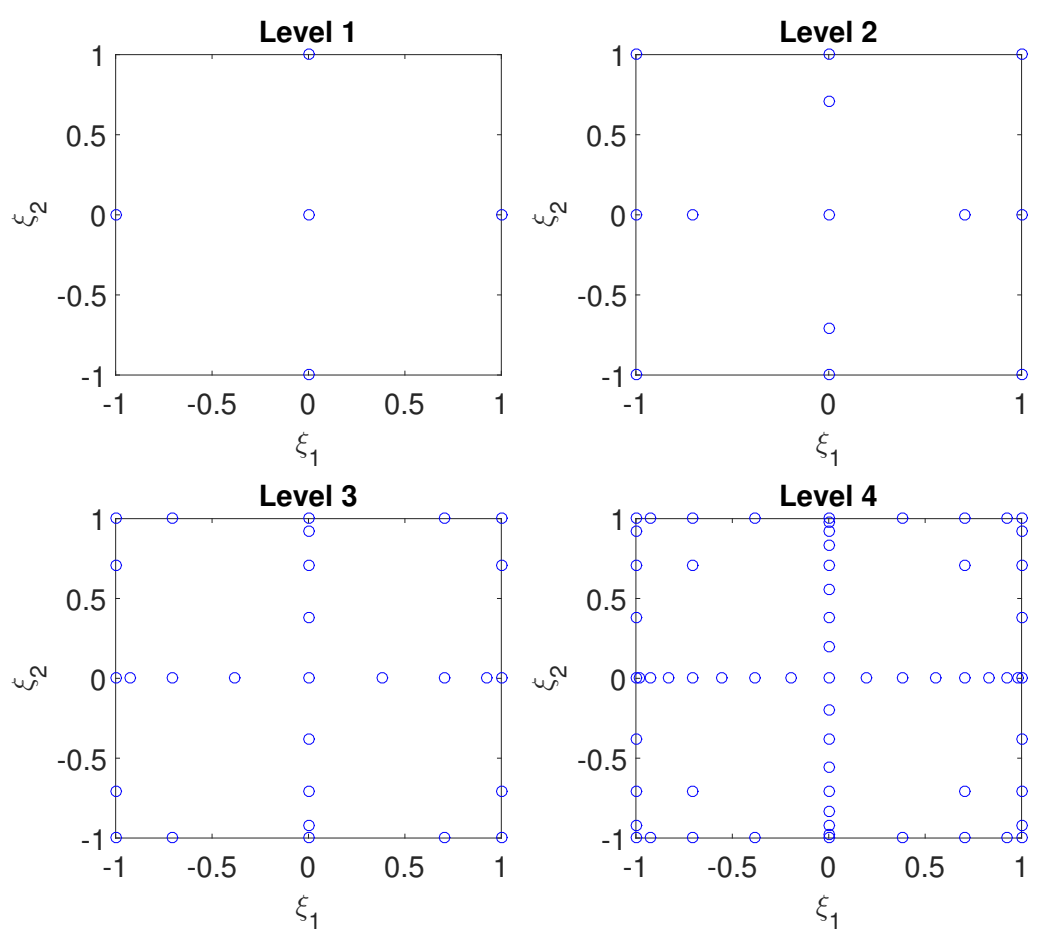

Figure 7: Clenshaw-Curtis Smolyak's nested Sparse Grids for different levels of accuracy.

\subsection{Sources of Uncertainty}

The sources of uncertainty in the present work are based on a mixture of outcomes of literature review and information extracted from experimentalists through critical analysis and discussions on the mechanical characteristics of a similar rotating pipe experimental facility at University of Málaga. The idea of this research is to conduct the propagation of uncertainties in the computational problem, with the view to improve the reliability of deterministic simulations. To define our sources of uncertainty, we referred to a set of relevant literature. [44] is a very related paper and motivation for this work, where a $2.5 \%$ of variance in uniform distribution in the swirl number and in the inlet velocity was applied, that means a $2.5 \%$ of variance in $Q$. In [74] a $\pm 3 \%$ of variation as uniform inlet velocity was applied. Also, in [75] velocity was measure within a $\pm 1 \%$ with the described measurement techniques. As a consequence of the mentioned reviews, a conservative value of uncertainty for $Q$ has been chosen as a $5 \%$ of variance. In addition, in the Fluid Mechanics laboratory at University of Málaga, the mechanical tolerance of the pipe angular velocity 
measurement was found to be $0.5 \%$. We have set this uncertainty for the angular velocity of the simulated pipe. Despite the nozzle-to-plate distance $H / D$ could be another parameter subject to uncertainty, $H / D$ usually exhibits very low uncertainty in experiments. Thus, it has been observed in [9] that around $H / D=5$, a variation of a $1 \%$ in $H / D$ would be less than 1 unit of variation in both the Nusselt number at the stagnation point and Nusselt surface averaged value. This, together with the emerging difficulties in generating a new mesh for each $H / D$ sample, lead us to exclude $H / D$ as random input.

Let $\bar{Q}$ and $\bar{\Omega}$ denote the deterministic mean values of the two uncertain parameters $Q$ and $\Omega$. Within this framework, and being conservative with respect to the literature results aforementioned, the source of uncertainties have been determined as the uniform distributions $Q \sim$ $\operatorname{Unif}(0.95 \bar{Q}, 1.05 \bar{Q})$ and $\Omega \sim \operatorname{Unif}(0.995 \bar{\Omega}, 1.005 \bar{\Omega})$. The main motivation in modelling the inputs by this type of distribution is conservativeness. The same probability is assigned to every value in the ranges of the variables, so it is ensured that the worst performance scenario is analysed. This approach has been used in the literature when insufficient information about experimental uncertainty is available, as e.g. in [44, 76]. In [76] a study of the impact of both Gaussian and uniform distributions was developed in CFD simulations of the flow over an airfoil. In our work, in order to observe the response to other distributions, truncated Gaussian distributions were also input in Section 7.2, besides the uniform distributions.

The advantage of dealing with $Q$ as stochastic input is that several uncertainties such as those related to measurement tools tolerance, pipe diameter, loss of pressure or density variations can be accounted in only one parameter. This also supports the idea of being conservative. If one wants to study each parameter separately, the dimension of the stochastic space would be unnecessarily increased and correlation-based sampling techniques must be used. That is, both the cost and difficulty would increase. In this work, there is no correlation amongst the chosen random variables, and this is important because the SCM applied in this work assumes independence amongst variables.

\section{Coupling of the Computational Simulations}

Since the turbulence closure used to model the pipe flow and the one used for the impinging heat transfer problem are both different, a single CFD simulation of the whole system cannot be developed. Moreover, the computational cost of the problem would be unnecessarily increased if the computed duct is long enough to ensure a fully-developed flow, resulting to an unnecessarily expensive domain to simulate.

To overcome this problem, two-step CFD simulations are coupled in the following way: Firstly, the CFD simulation of the swirling flow confined in a rotating pipe solved with the RSM turbulent model are completed (Simulation 1). Secondly, both the velocity and some turbulent dimensionless profiles at the exit of the pipe are input as inlet boundary conditions for the heat transfer simulation (Simulation 2). In particular, the turbulent parameters are defined by

$$
k=\frac{2}{3}(U I)^{2}
$$




$$
\omega=\rho \frac{k}{\mu}\left(\frac{\mu_{t}}{\mu}\right)^{-1}=\rho \frac{k}{\mu} \beta^{-1},
$$

where $k$ is the turbulent kinetic energy, $I$ is the turbulence intensity, $U$ is the time-average velocity of the flow, $\rho$ is the density of the fluid, $\omega$ is the specific turbulent dissipation rate and $\mu_{t} / \mu \equiv \beta$ is defined as the turbulent viscosity ratio, represented by $\beta$ throughout this paper. The turbulent kinetic energy is available from the RSM simulations, and the turbulent dissipation rate can be evaluated by Eq. (9) prior to running Simulation 2.

In order to couple the two simulations for the modelling, two options have been tested in this work for Simulation 2, as shown in Fig. 8. The first one is to impose the simulated profiles at the exit of the rotating pipe in Simulation 1 as inlet conditions onto Simulation 2, for each required deterministic simulation for uncertainty quantification. The second option is to develop non-linear regression models for the fully-developed swirling turbulent flow profiles emerging from the pipe, avoiding to compute Simulation 1. To avoid the simulation of the rotating pipe would be then possible because the regression profiles introduced in Section 6 can be directly input as parametric functions onto Simulation 2 by means of a User-Defined Function (UDF). The definitive parametric regression profiles are only dependent on the parameters $Q$ and $\Omega$, which are CFD input parameters. These new profiles are imposed in the inlet boundary condition of Simulation 2 as profiles for velocity (axial and azimuthal velocity profiles) and turbulence (turbulent viscosity ratio and turbulent kinetic energy profiles). The differences between these approaches will be analysed in this work to test whether these provide equivalent results in the heat transfer by impinging jets. If swirling jet flow profiles with/without uncertainty estimates are required for other applications (for instance, for a plate with variations on its surface [7]), the use of these empirical models would avoid to simulate the rotating pipe flow.

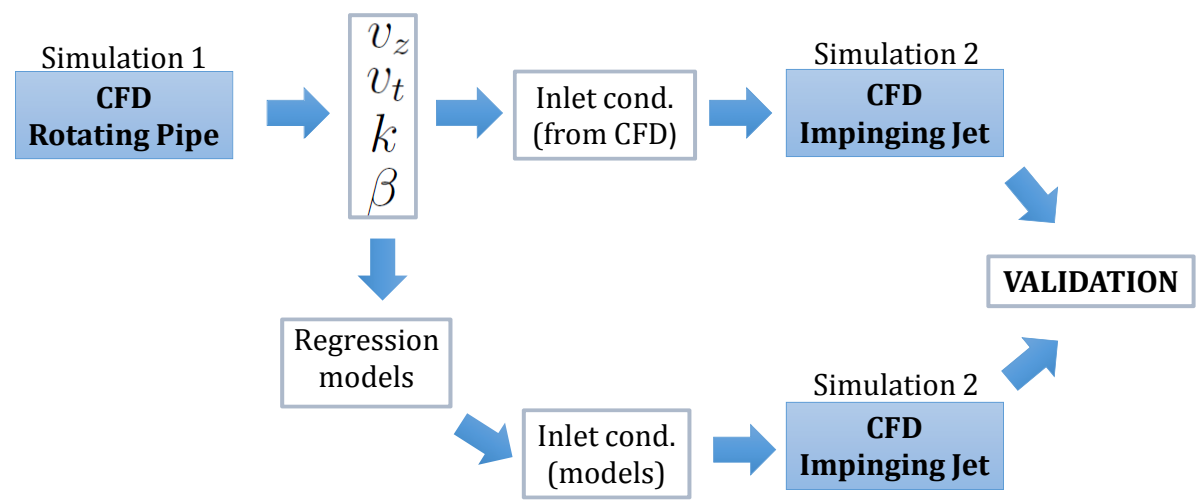

Figure 8: Two alternatives for conducting Simulation 2: using the CFD profiles at the exit of the nozzle or to impose mathematical models. 


\section{Modelling of Dimensionless Profiles From the Rotating Pipe Flow of Simulation 1}

To quantify the uncertainty of the output of Simulation 2, several profiles should be systematically generated from Simulation 1 and then be input as boundary condition into Simulation 2. In Section 7.1, SCM is applied $r / R$ point by $r / R$ point to the output profiles from Simulation 1. Then, in Section 7.2, the uncertainty in the Nusselt number is quantified. Despite the fact that SCM provides a $Q-\Omega$ dependent response surface for the profiles, the dependence of these profiles on the spatial coordinate $r / R$ makes inefficient to code hundreds of surrogates in the UDF, one for each $r / R$ point.

Another option to compute uncertainty could be to include $r / R$ as an additional parameter of the stochastic space. This would be even more cumbersome, because the number of collocation points (CFD simulations) would be dramatically increased to deal with this new parameter of high non-linearity in the profiles. The suggested approach by means of non-linear regression models overcomes both problems, providing easy functions to code in any CFD software. The differences between the direct SCM approach and the use of the models is investigated in this work.

This type of surrogate model has been studied in [16] and successfully implemented in [7, 11, 12]. The use of such modelling is in fact useful in industrial and academic applications, and one can find this, e.g. in stability analysis of jets flows as in $[65,66]$, where researchers used empirical functions of jet flows from [61, 62, 63, 64] to test their approaches.

In the investigation of the non-linear regression models, a Reynolds number of $R e=23000$ is considered. Despite the modelling may seem focused on a particular application, this Reynolds number is one of the most studied test cases in the literature with/without swirl, e.g. in [14, 58, 59, $31,26,60,18,27,9,13,25]$. Thus, the proposed models for the swirling flow under uncertainty can be directly input as boundary condition to other investigations with similar set-up such as systems with arrays of impinging jets [77, 78], swirling jets impinging on a plate with bumps/dimples [7], or swirling turbulent flows in an axisymmetric sudden expansion [79, 44], amongst others. The computational efforts to perform UQ/CFD on these new applications would be only restricted to a single simulation. It is also relevant to mention that an analytical solution based on physics ground was not considered and this is the main limitation of this approach, as the flow is turbulent and this makes a pure mathematical analysis not feasible.

In the present study, four dimensionless models are given: axial velocity $\left(v_{z}\right)$, azimuthal velocity $\left(v_{t}\right)$, turbulent kinetic energy $(k)$ and turbulent viscosity ratio $(\beta)$ profiles. These models approximate the response of the CFD RANS profiles for the different $Q$ and $\Omega$, although initially introduced in this section for the deterministic base case $(R e=23000$ and $S=1)$. In the literature one can find often that inlet boundary conditions for turbulence quantities are modelled as a percentage (turbulence intensity), as in [44, 74, 80]. Therefore, to provide specific profiles for both the turbulent kinetic energy and viscosity ratio instead of broad percentages, intends a more accurate insight.

These models are composed of several functions chosen according to the features of the profiles and trained against data from Simulation 1. Interpolation/splines fit was considered, however, 
a new model should be built for each CFD simulation profile, not being a good alternative to model a link between the profiles and $Q$ and $\Omega$. For this reason, a non-linear parametric regression approach for the profiles plus a polynomial regression for the coefficients is preferred, as this approach makes possible to build profiles dependent on both the physical parameters $Q$ and $\Omega$ under uncertainty. These coefficients are found by a MATLAB custom code which calls Curve Fitting Toolbox functions that use a Non-Linear Square method with Trust-Region algorithm [81]. The introduced piecewise-like process to fit these models can be reproduced by other researchers in $\mathrm{CFD} /$ experimental profile modelling purposes.

Several parametric models were tested for the axial and azimuthal radial velocities as shown in Fig. $9^{2}$. For the axial velocity radial profile, it can be observed that the profile shape is nonlinear with different piecewise curvatures. It is specially relevant for the proper modelling of the boundary layer, since this plays an important role in the further development of the flow. This led to select a parametric function with a hyperbolic tangent for $v_{z} / U^{(1)}, v_{z} / U^{(2)}, v_{z} / U^{(b e s t)}$. This is consistent with the past work of $[82,16]$ that reports boundary layer of flows under rotation having a hyperbolic tangent shape. In jet flows this feature has also been observed since a hyperbolic tangent profile takes place when two flows are in contact with different but uniform axial velocities [83]. As the flow is turbulent and with swirl, the axial velocity radial profile differs from the classic parabolic profile of a Hagen-Poiseuille flow. Nevertheless, this modelling was also tried with a cubic polynomial in $v_{z} / U^{(3)}$, which requires only three constants to estimate, but had a poor performance. Amongst $v_{z} / U^{(1)}$ and $v_{z} / U^{(2)}$ there are some clear differences. A cubic power factor in $v_{z} / U^{(1)}$ is introduced to deal with the non linearity, which is not introduced in $v_{z} / U^{(2)}$, undergoing a bad fit. The combination of the power function featured in $v_{z} / U^{(1)}$ with the flexibility of the third order polynomial $v_{z} / U^{(3)}$ led to the selected model in Eq. (10), the $v_{z} / U^{(\text {best })}$, which performs the best fit among the tested ones.

The procedure to model the azimuthal velocity profile was simpler. It was already shown in [17] that this radial profile is nearly parabolic. However, an exponential constant was also tried. In Fig. 9, it is shown that the power function fitted the best, and the predicted values of the constants are $a_{t}=0.9709$ and $b_{t}=2.0052$, thus $v_{t} / U \sim(r / R)^{2}$.

The modelling of $k / U^{2}$ was the most complicated parametric regression because of the less smooth changes in slope. Four different regression models were tested, as shown in Fig. 10. The best function for the region near to the wall was the hyperbolic tangent. $k / U^{2(2)}$ did not have a bad fit in that region without that term, however, its fit was not good for the rest of the profile. Thus, efforts were focused on improving the exponential term of $k / U^{2(1)}$, consistently to its shape. As shown in Fig. 10, $k / U^{2(3)}$ is far from the training points.

The coefficients were found to be difficult to regress using the MATLAB curve fitting algorithm. The reason may be that the algorithm struggles to fit the $k / U^{2}$ data with four coefficients, being required to reduce the dimension in the search space. An accurate initial guess may solve this issue, but even with the $k / U^{2(b e s t)}$ coefficients as initial guess, the fitting search was not possible. In $k / U^{2(3)}$, it was observed that the $a$ coefficient tended to a value close to 0.05327 . Despite the fact that the fitting is very accurate, to fix the 0.05327 and 1.2 coefficients in the equation is reducing

\footnotetext{
${ }^{2}$ In this manuscript the superscript $(i)$ denotes the i-th fitted profile, and (best) stands for the best fitting function amongst the tested ones.
} 


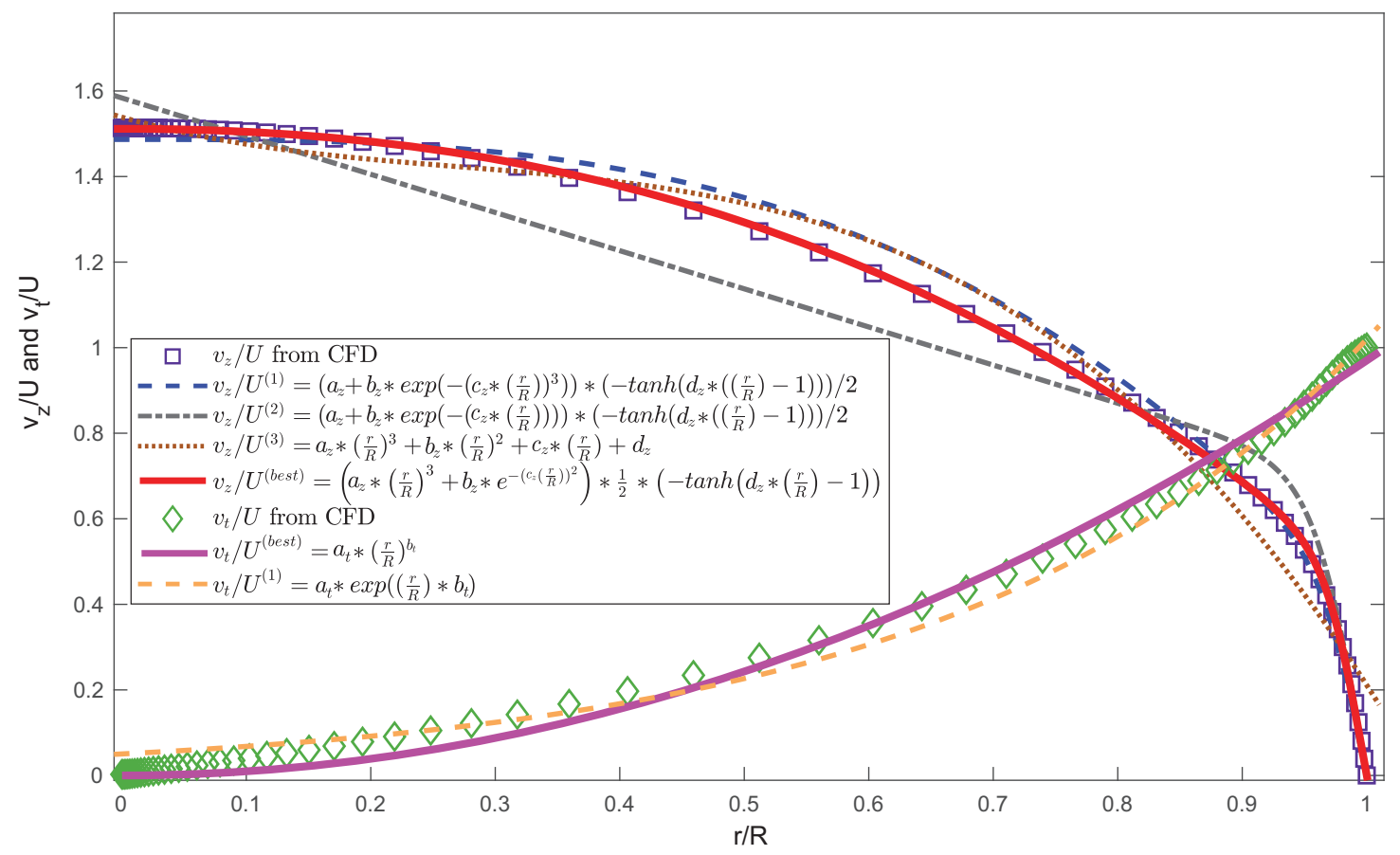

Figure 9: Axial and azimuthal velocity profiles from CFD and the non-linear regression models for $R e=23000$ and $S=1$.

the flexibility of the model to adapt to changes in $Q$ and $\Omega$.

The turbulent viscosity ratio, $\beta$, was also modelled with four regression models and their performance can be seen in Fig. 11. An exponential decay was tested, $\beta^{(1)}$, which reproduces well the shape of the profile. The power function was suitable to model the curvatures. Since the shape in the intermediate region is similar to the shape observed in the axial velocity profile, the same power functions were tried with a hyperbolic tangent in $\beta^{(2)}$. But unfortunately the fit close to the wall was fully linear, with no curvature approaching $\beta=0$. This led to testing the product of exponential functions in $\beta^{(3)}$. Some difficulties in fitting the $r / R<0.8$ region were noticed and the addition of a coefficient $d$ in the power of the left hand exponential solved that successfully. In order to reduce the dimension in the search space, the $d$ coefficient in $\beta^{(3)}$ was fixed to 13 , as it mainly controls the curvature approaching $\beta=0$.

After fitting the non-linear regression models to the CFD data profiles, the proposed ones are those labelled as best in Figs. 9-11:

$$
\frac{v_{z}}{U}=\left(a_{z}\left(\frac{r}{R}\right)^{3}+b_{z} e^{-\left(c_{z}\left(\frac{r}{R}\right)\right)^{2}}\right) \frac{1}{2}\left(-\tanh \left(d_{z}\left(\frac{r}{R}\right)-1\right)\right)
$$




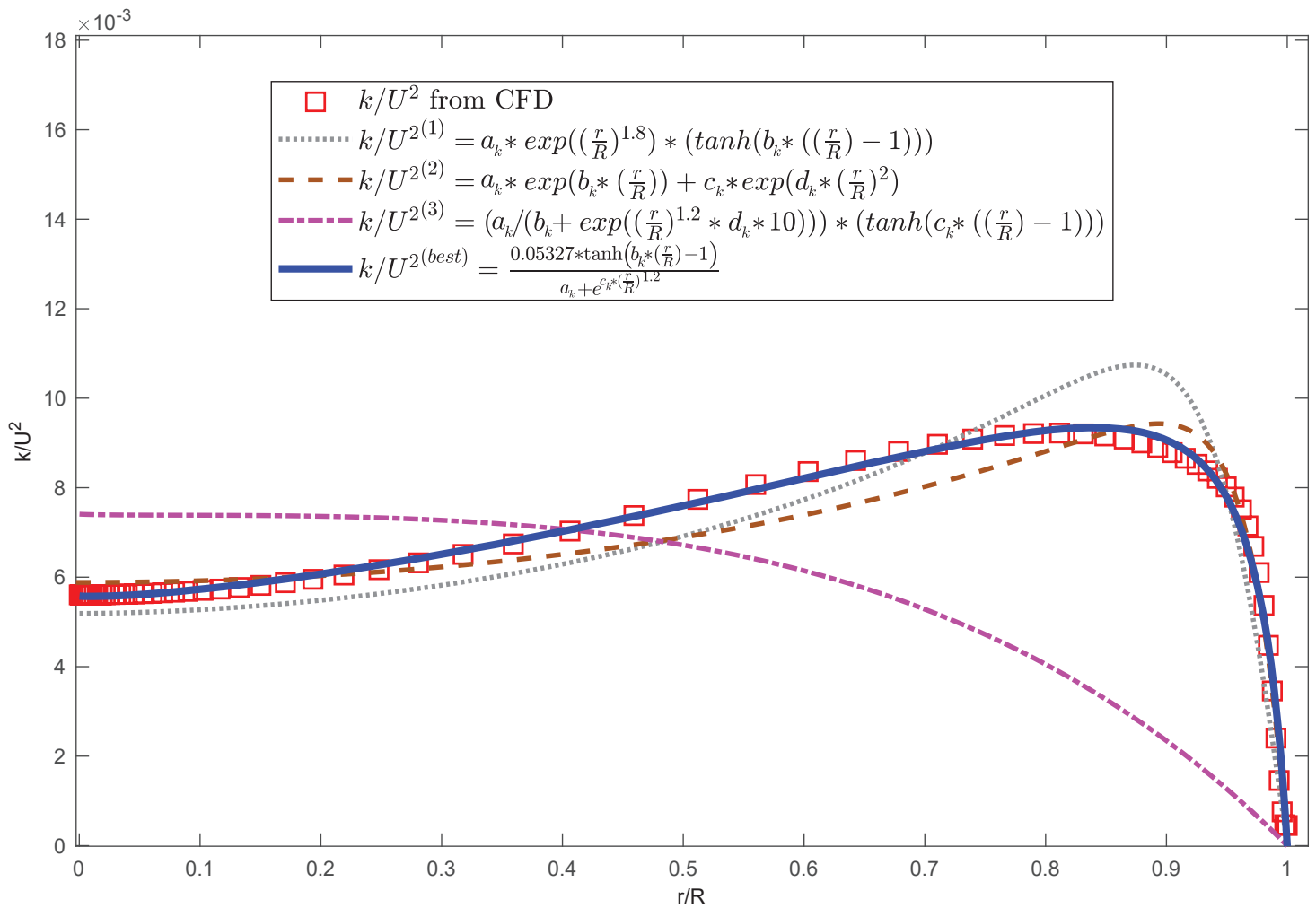

Figure 10: Dimensionless turbulent kinetic energy profile from CFD and the non-linear regression models for $R e=23000$ and $S=1$.

$$
\begin{gathered}
\frac{v_{t}}{U}=a_{t}\left(\frac{r}{R}\right)^{b_{t}}, \\
\frac{k}{U^{2}}=\frac{0.05327 \tanh \left(b_{k}\left(\frac{r}{R}\right)-1\right)}{a_{k}+e^{c_{k}\left(\frac{r}{R}\right)^{1.2}}} \\
\beta=a_{\beta} e^{-b_{\beta}\left(\frac{r}{R}\right)^{d_{\beta}}}\left(e^{-c_{\beta}\left(\left(\frac{r}{R}\right)^{13}-1\right)}-1\right) .
\end{gathered}
$$

The coefficients of the mathematical models, addressed for sake of notation as $\gamma_{i}$, where $\gamma=$ $a, b, c, d$ and $i=z, t, k, \beta$, are shown in Table 1. In Figs. 12-14, the goodness of fit is plotted by the $95 \%$ confidence prediction bound and goodness measures. The higher the Reynolds number at constant $S$ value, the less noticed the curvature of $v_{t} / U$ as seen in [9], so the experimental $v_{t} / U$ profile in Fig. 12 is expected to be closer to the simulated and modelled profiles. The model with the widest confidence interval is $k / U^{2}$. The model for $v_{t} / U$ has a notable prediction bound as well, which likely results from the relatively simple formulation (only two unknown coefficients). A more complex parametric function would be required to capture the negative curvature shown by the CFD data over the range $0.95<r / R<1$. 


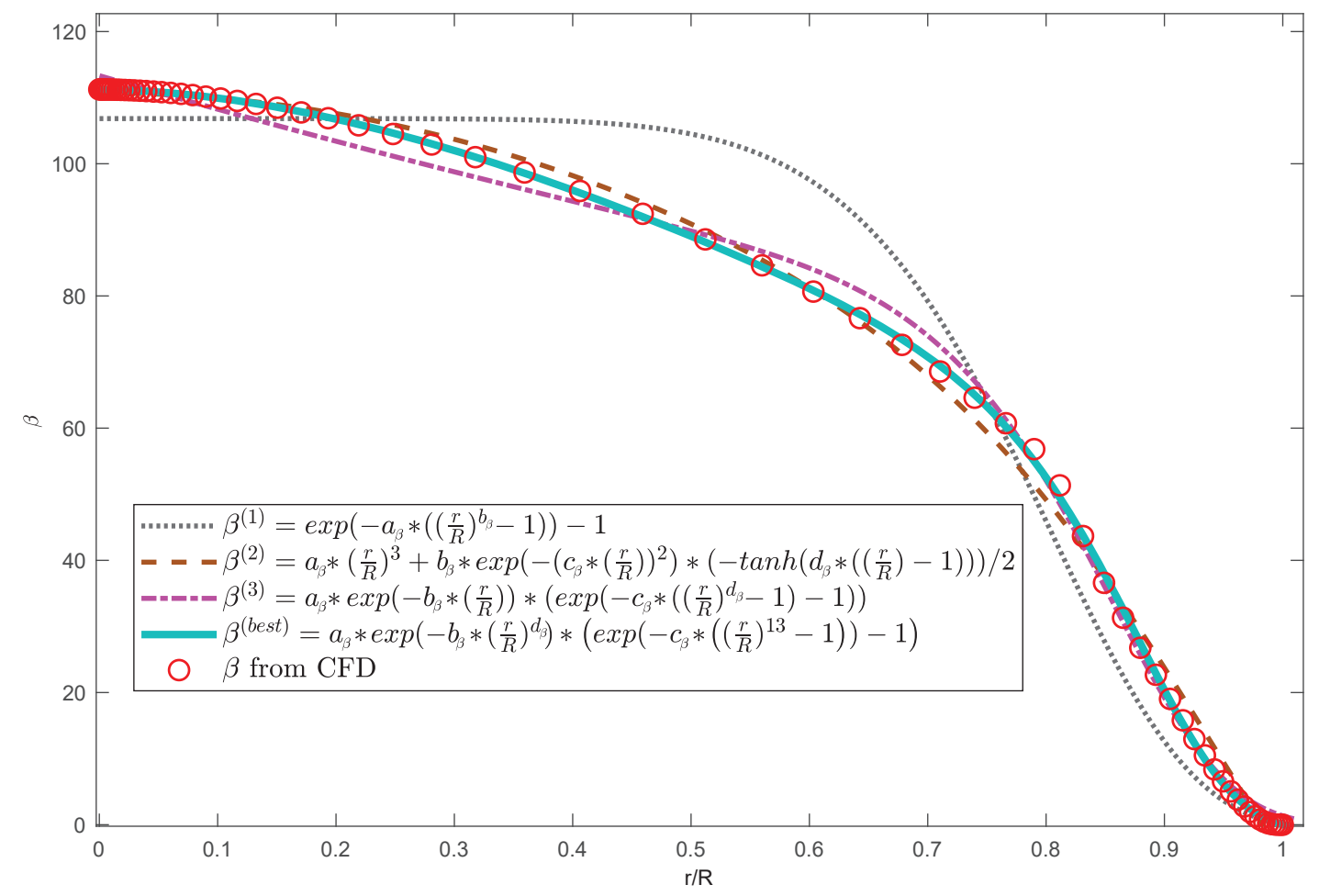

Figure 11: Viscosity ratio profile and the non-linear regression models for $R e=23000$ and $S=1$.

It is important to ensure that the regression coefficients are enclosed within a short confidence bound, which denotes a good fit. The confidence bounds for the regression coefficients are calculated as

$$
c_{b}=\gamma_{i} \pm t_{0.95, v} S E_{\gamma_{i}},
$$

where $t_{0.95, v}$ refers to the two-tailed value of the inverse of t-Student distribution for $v$ degrees of freedom and a $95 \%$ level of confidence, and $S E_{\gamma_{i}}$ refers to the estimated standard error of $\gamma_{i}$. The results can be seen in Table 1 for the deterministic base case $(R e=23000$ and $S=1)$. These values, as well as the goodness of fit metrics in Table 2, were monitored for all the fittings of the collocation points when studying the propagation of uncertainty, showing almost identical performance. In Table 2 it can also be found the goodness indicators of the fit for the deterministic base case. As the coefficient of determination (defined in this manuscript as $\hat{R}^{2}$, to avoid confusion with $R$, the radius of the pipe) is not the best measure for the goodness of a fit in non-linear responses or with overfitting, the Sum of Squares due to Error (SSE), Adjusted- $\hat{R}^{2}$ and Root Mean Squared Error (RMSE) are also given. When increasing the number of terms in the models, $\hat{R}^{2}$ increases, despite of the model can be overfitted. It is hence important to consider the Adjusted- $\hat{R}^{2}$, whose value in case of overfitting starts to decrease.

In the coefficient estimation process described above, the regression models do not depend on 
Table 1: Fitting coefficients of the dimensionless profiles for the deterministic base case $(R e=$ 23000 and $S=1)$.

\begin{tabular}{c|cc}
\hline Coefficient & Value, $\gamma_{i}$ & $95 \%$ confidence bound, $c_{b}$ \\
\hline$a_{z}$ & -1.0474 & $(-1.1231,-0.9718)$ \\
$b_{z}$ & 3.0230 & $(3.0169,3.0291)$ \\
$c_{z}$ & -0.6538 & $(-0.6765,-0.6310)$ \\
$d_{z}$ & 34.1414 & $(32.6899,35.5929)$ \\
\hline$a_{t}$ & 0.9709 & $(0.9563,0.9854)$ \\
$b_{t}$ & 2.0052 & $(1.9048,2.1056)$ \\
\hline$a_{k}$ & -0.1708 & $(-0.1815,-0.1602)$ \\
$b_{k}$ & -0.0880 & $(-0.0890,-0.0870)$ \\
$c_{k}$ & -1.6985 & $(-1.7538,-1.6432)$ \\
\hline$a_{\beta}$ & 1.9314 & $(1.7141,2.1486)$ \\
$b_{\beta}$ & 0.7933 & $(0.7712,0.8154)$ \\
$c_{\beta}$ & 4.0472 & $(3.9363,4.1581)$ \\
$d_{\beta}$ & 1.8621 & $(1.8048,1.9194)$ \\
\hline
\end{tabular}

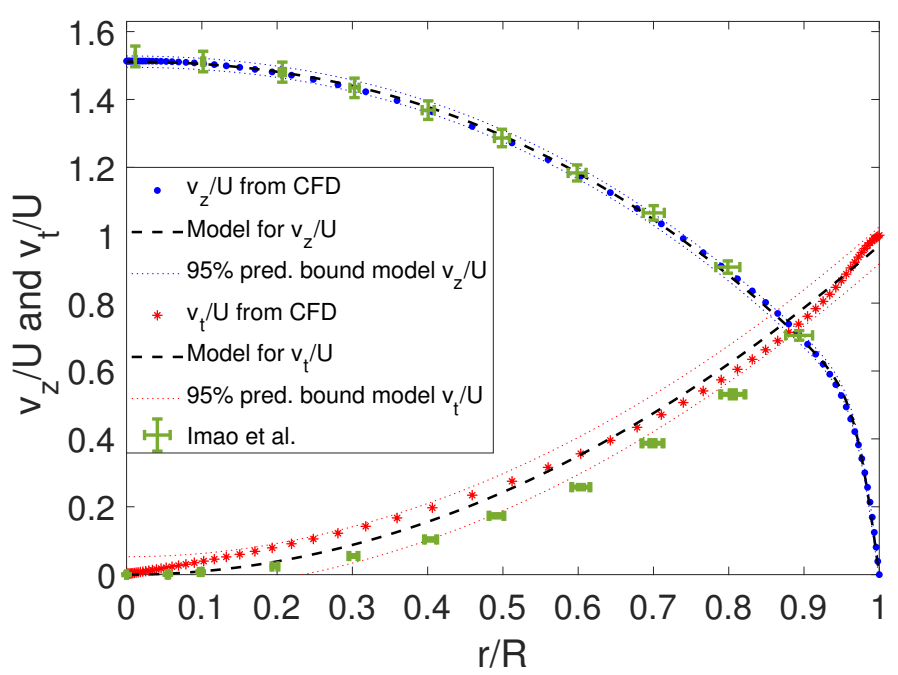

Figure 12: Axial and azimuthal velocity profiles from CFD and non-linear regression model fits with prediction bounds for $R e=23000$ and $S=1$. Imao et al. are the experimental data from [17] with reported error bars of a $2 \%$.

$Q$ and $\Omega$. Therefore, Eqs. (10)-(13) are written as

$$
\left(v_{z} / U, \quad v_{t} / U, \quad k / U^{2}, \quad \beta\right)^{t}=\mathbf{F}\left(\gamma_{i}\right) .
$$

To turn Eqs. (10)-(13) into $Q$ and $\Omega$ dependent functions, polynomial regression models can 


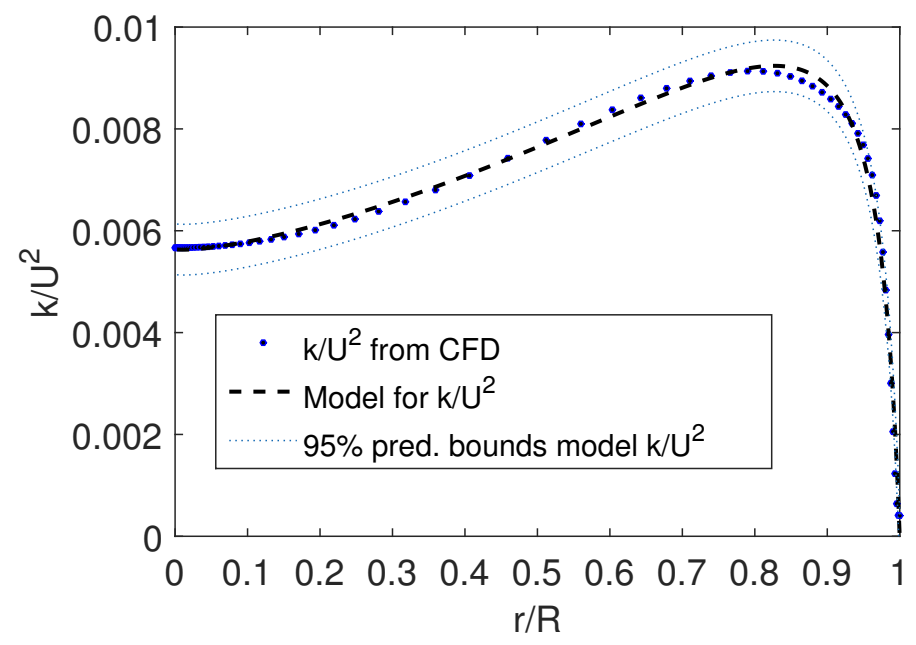

Figure 13: Dimensionless kinetic energy profile from CFD simulations and non-linear regression model fits with prediction bounds for $R e=23000$ and $S=1$.

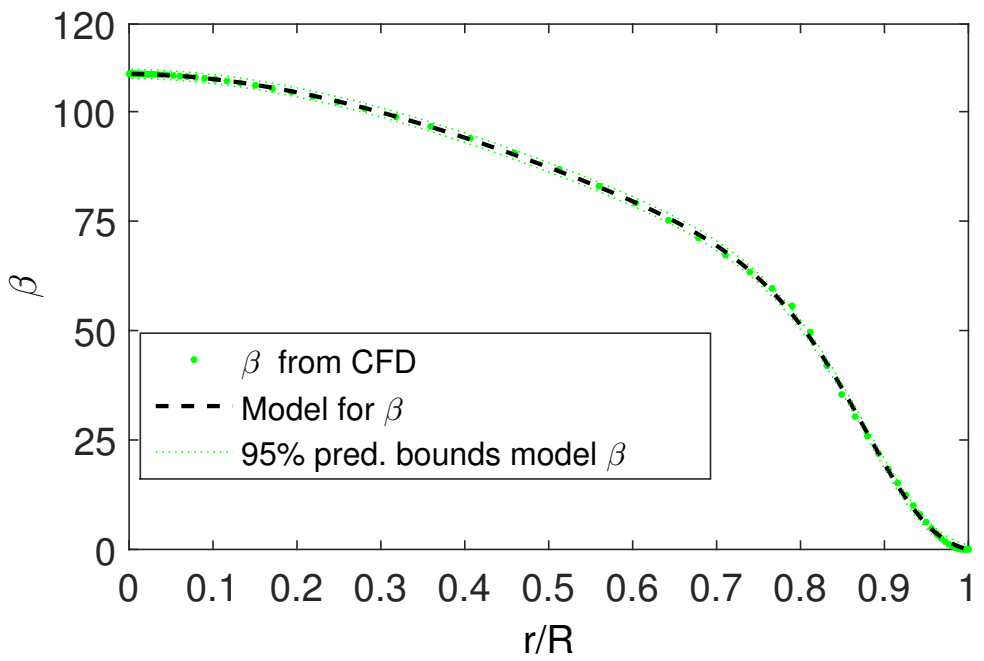

Figure 14: Turbulent viscosity ratio profile from CFD simulations and non-linear regression model fits with prediction bounds for $R e=23000$ and $S=1$.

be built as

$$
\left(v_{z} / U, \quad v_{t} / U, \quad k / U^{2}, \quad \beta\right)^{t}=\mathbf{F}\left(\gamma_{i}\right)=\mathbf{G}(Q, \Omega)
$$

As a consequence of this approach, the suggested models for the profiles are linked to the input random variables. These polynomial fits are shown in Appendix A, with the corresponding equations, goodness of fit and plots. In addition to this, in Appendix B the User Defined Function (UDF) for its implementation in FLUENT is given.

The domain of Eq. (16) for the uncertainty analysis is constrained to $Q \in[0.95 \bar{Q}, 1.05 \bar{Q}]$ and $\Omega \in[0.995 \bar{\Omega}, 1.005 \bar{\Omega}]$, which is set by the domain of the uniform input uncertainties introduced in 
Table 2: Goodness of fit for the non-linear regression model profiles for the deterministic base case $(R e=23000$ and $S=1)$.

\begin{tabular}{c|cccccc}
\hline Profile & SSE & $\hat{R}^{2}$ & Adjusted- $\hat{R}^{2}$ & RMSE & $m$ & $v$ \\
\hline$v_{z} / U$ & 0.0044 & 0.9997 & 0.9997 & 0.0082 & 4 & 65 \\
$v_{t} / U$ & 0.0536 & 0.9947 & 0.9946 & 0.0283 & 2 & 67 \\
$k / U^{2}$ & $3.9766 \mathrm{e}-06$ & 0.9865 & 0.9861 & $2.4546 \mathrm{e}-04$ & 3 & 66 \\
$\beta$ & 16.6794 & 0.9999 & 0.9999 & 0.5066 & 4 & 65 \\
\hline
\end{tabular}

Section 4.2. The performance of the proposed models for larger values of $S$ (and therefore, $\Omega$ ), can be seen in Fig. 15(a)-(c). In these plots, it is shown a comparison between the CFD and modelled profiles with a very good match. In these figures it is observed that $S$ can be varied at least from 0 to 1, with $Q$ fixed at its deterministic base value, and these fitting results provide a promising fit [2]. Amongst the studied profiles, the turbulent kinetic energy develops a near-wall peak that is not rendered by the current fit. Further numerical models can be investigated to suitably model this feature, which is out of scope in this work. The coefficients of these fittings are provided in Figs. 16 and 17.

\section{Uncertainty Quantification Results of the Two-Step CFD Simulation.}

\subsection{Simulation 1. Uncertainty Quantification of the Fully-developed Turbulent Swirling Flow Generated by the Rotation of a Pipe}

Regarding the uncertainty quantification process, in Section 4 the Stochastic Collocation method has been introduced and the results of analysing Simulation 1 are shown in Table $3 \& 4$. It can be observed that even using few points for the C-C Sparse Grid, the statistical moments are not changing remarkably. This is because despite of the non-linear behaviour behind the equations that govern the problem, the outputs of interest have a linear response with respect to the ranges of the random inputs in the simulation of the pipe. A budget (Design of Experiment, DoE) of few collocation points was enough, as increasing the number of collocation points there is no change, as expected (see Table $3 \& 4$ ). However, the budget of 65 collocation points was necessary to monitor the convergence of the stochastic variance of the Nusselt number in Simulation 2, and therefore, 65 profiles for each velocity and turbulent parameter are required from Simulation 1 when the mathematical models coded in the UDF are not used as substitute.

The outputs of interest from Simulation 1 are the dimensionless velocity and turbulent profiles at the exit of the pipe, as the pipe becomes the nozzle for the impinging problem and these profiles will be used as inlet boundary conditions. The uncertainty analysis of these profiles can be seen in Fig. 18 and 19, where the mean and standard deviation envelopes of those profiles are shown. Experimental data was only available for the velocity profiles, used to validate the CFD 


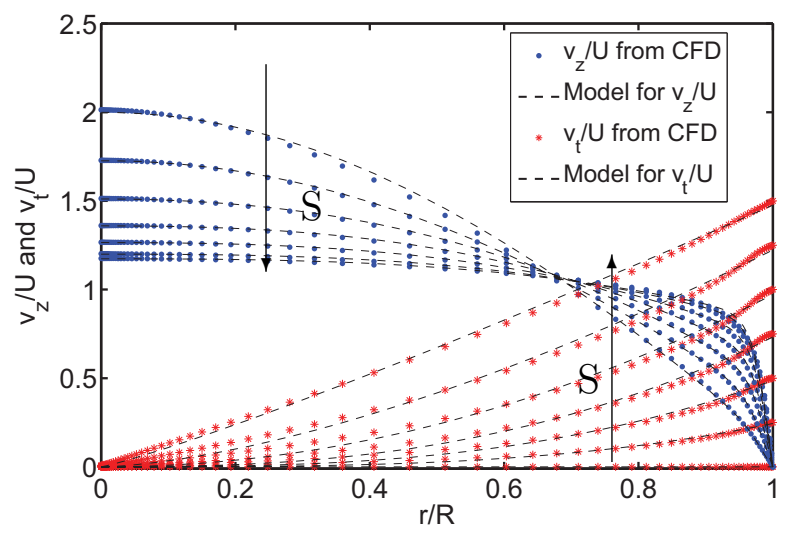

(a)

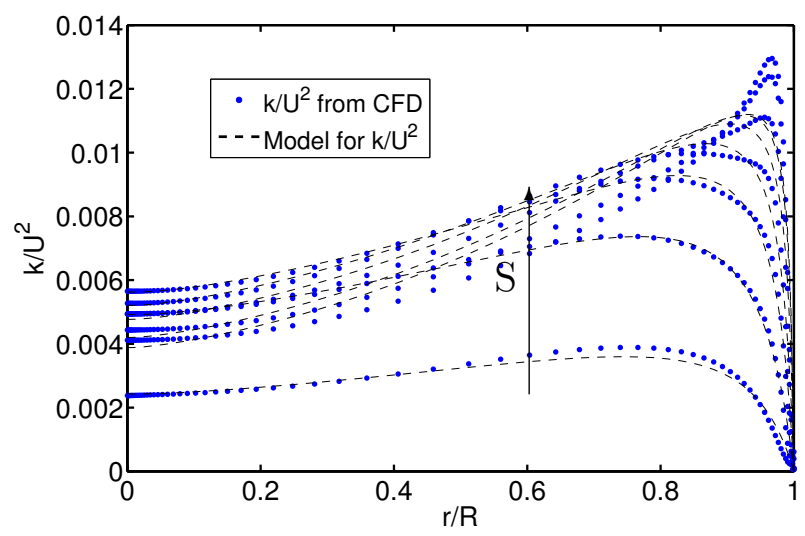

(b)

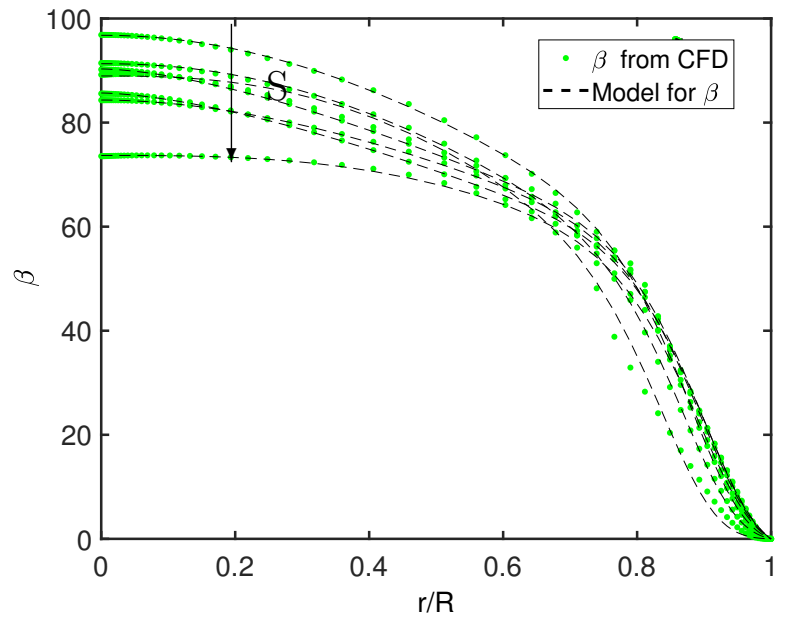

(c)

Figure 15: Dimensionless profiles from CFD and their suggested models for (a) axial and azimuthal velocity, (b) turbulent kinetic energy and (c) turbulent viscosity ratio, for $S$ varied from 0 up to 1.5.

simulations in [9] and also included in Fig. 18. The plots show that for the values of $Q$ and $\Omega$ 

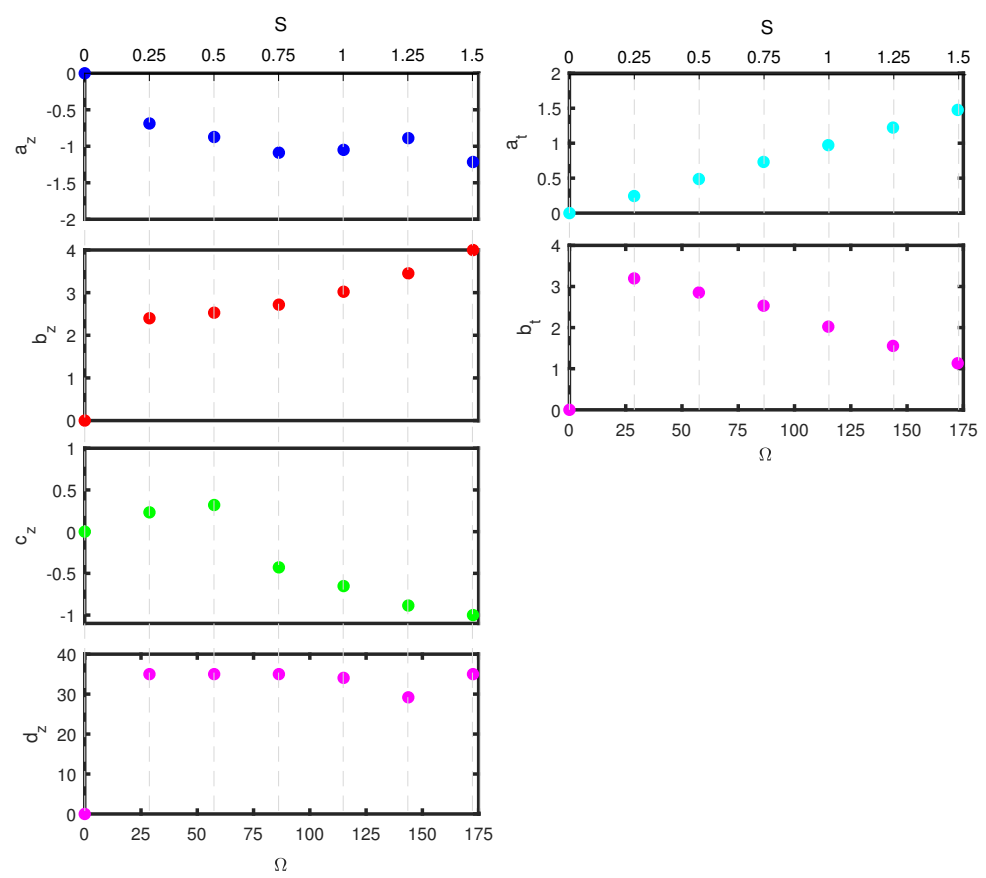

Figure 16: Coefficients for the dimensionless velocity profiles in Figure 15.

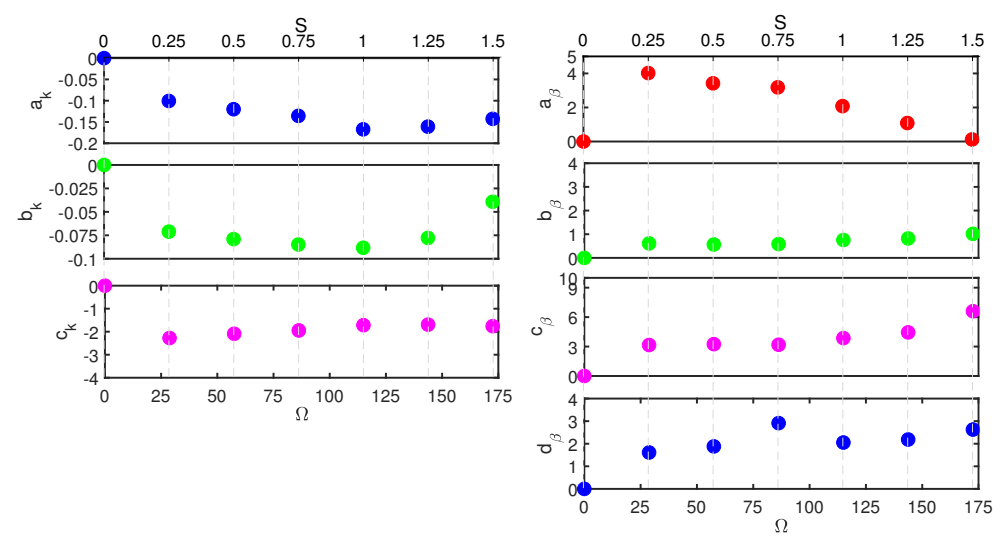

Figure 17: Coefficients for the dimensionless turbulent profiles in Figure 15.

\begin{tabular}{cccccc}
\hline Level & Points & $\lambda_{r / R=1}$ & $I(\%)_{r / R=0.5}$ & $\left(v_{z} / U\right)_{r / R=0.5}$ & $\left(v_{t} / U\right)_{r / R=0.5}$ \\
\hline 1 & 5 & 0.01373401 & 8.45783368 & 1.28350388 & 0.26677295 \\
2 & 13 & 0.01373399 & 8.45774137 & 1.28350593 & 0.26676999 \\
3 & 29 & 0.01373404 & 8.45773429 & 1.28350567 & 0.26676957 \\
4 & 65 & 0.01373401 & 8.45773957 & 1.28350436 & 0.26676961 \\
\hline
\end{tabular}

Table 3: Stochastic means of the friction factor $(\lambda)$, turbulent intensity $(I)$, dimensionless axial $\left(v_{z} / U\right)$ and azimutal $\left(v_{t} / U\right)$ velocity at $r / R=0.5$, at the exit of the rotating pipe. 


\begin{tabular}{cccccc}
\hline Level & Points & $\lambda_{r / R=1}$ & $I(\%)_{r / R=0.5}$ & $\left(v_{z} / U\right)_{r / R=0.5}$ & $\left(v_{t} / U\right)_{r / R=0.5}$ \\
\hline 1 & 5 & $0.22152010 \mathrm{e}-06$ & $0.72630289 \mathrm{e}-03$ & $0.75705236 \mathrm{e}-04$ & $0.22938667 \mathrm{e}-03$ \\
2 & 13 & $0.22059469 \mathrm{e}-06$ & $0.72520829 \mathrm{e}-03$ & $0.75256672 \mathrm{e}-04$ & $0.22639809 \mathrm{e}-03$ \\
3 & 29 & $0.22066632 \mathrm{e}-06$ & $0.72553382 \mathrm{e}-03$ & $0.75245877 \mathrm{e}-04$ & $0.22636540 \mathrm{e}-03$ \\
4 & 65 & $0.22067550 \mathrm{e}-06$ & $0.72554659 \mathrm{e}-03$ & $0.75261365 \mathrm{e}-04$ & $0.22636600 \mathrm{e}-03$ \\
\hline
\end{tabular}

Table 4: Stochastic variances of the friction factor $(\lambda)$, turbulent intensity $(I)$, dimensionless axial $\left(v_{z} / U\right)$ and azimutal $\left(v_{t} / U\right)$ velocity at $r / R=0.5$, at the exit of the rotating pipe.

ranging within the considered uncertainty source intervals, the variance in the profiles is relatively small, as evidenced by the modest width between the dashed lines. Fig. 18 shows that the CFD predictions of the axial velocity perfectly match with experimental data, but also the reported experimental uncertainty. Regarding the azimuthal velocity, we recall that the deterministic CFD simulation validated in [9] had a good match with the experimental data, but systematically overpredicted $v_{t} / U$. Despite this, the achieved simulation is very accurate, especially near the wall, where the azimuthal velocity is higher and has greater impact on the results. Whilst the reported experimental uncertainty in [17] is a $2 \%$, the propagated uncertainty in the simulation is around a $3-4 \%$, which is still very low and realistic, taking into account that the CFD simulation overpredicts the azimuthal component. So, overall, the standard deviation was not large, which means that the simulation of the pipe under uncertainty is not sensitive to the modelled random inputs, as experimental data from the literature also reported.

As these output uncertainties will be propagated to the next simulation, these may affect the further behaviour of the impinging jet. For this reason, the uncertainty analysis in Simulation 2 is developed in the next section.

\subsection{Simulation 2. Uncertainty Quantification on the Impinging Swirling Jet for Heat Transfer: Boundary Conditions from first-step CFD simulations.}

In Simulation 2, a heat transfer process from a heated flat plate to an impinging swirling jet takes place. For uncertainty quantification purposes, the same Stochastic Collocation method with the Clenshaw-Curtis nested rule with sparse grid quadrature points is used as in Simulation 1. In order to be consistent with the deterministic simulations from the rotating pipe for different values of $Q$ and $\Omega$, the same collocation points simulated in that previous step are systematically used.

In this framework, uncertainties are propagated from the input of Simulation 1 to the output of Simulation 2. The convective heat transfer is quantified by the Nusselt number $N u$ both at the stagnation point and its surface averaged value on the plate, denoted respectively by $N u_{0}$ and $N u_{\text {avg }}$, with the latter defined by

$$
N u_{\text {avg }}=\frac{1}{\pi R_{\text {int }}^{2}} \int_{0}^{R_{i n t}} N u(s) 2 \pi s d s,
$$

where $R_{\text {int }}$ is the radius of the impinged flat plate and its value is set to $7.5 D$. The stochastic mean 


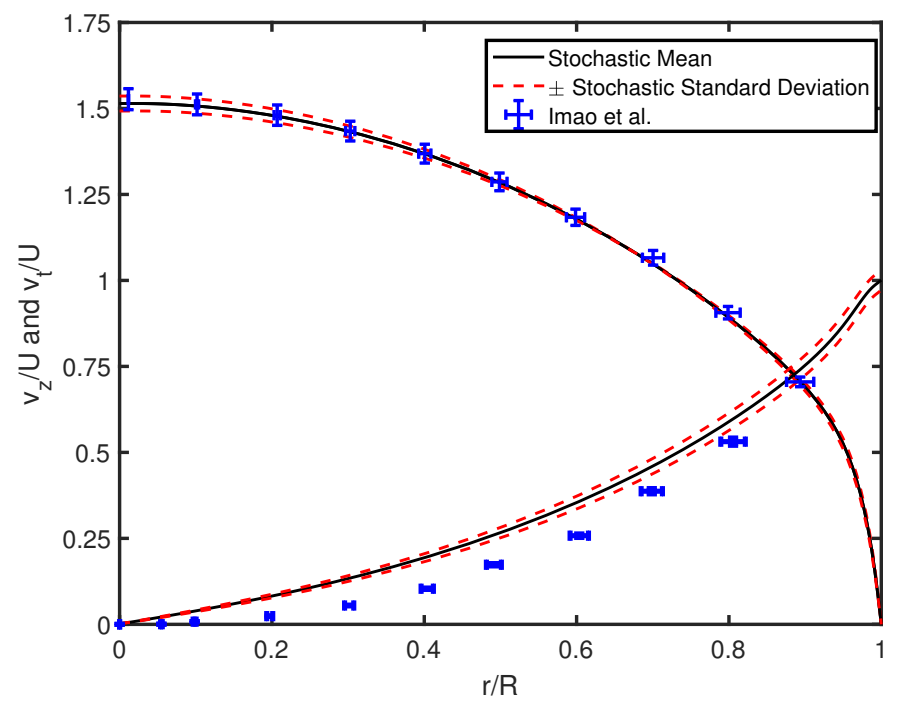

Figure 18: Radial distribution of the dimensionless axial and azimuthal velocity profiles at the exit of the pipe for the level 4 of the C-C Sparse Grid at $R e=23000$ and $S=1$. Imao et al. are the experimental data from [17], where $2 \%$ is reported as experimental uncertainty represented by error bars.
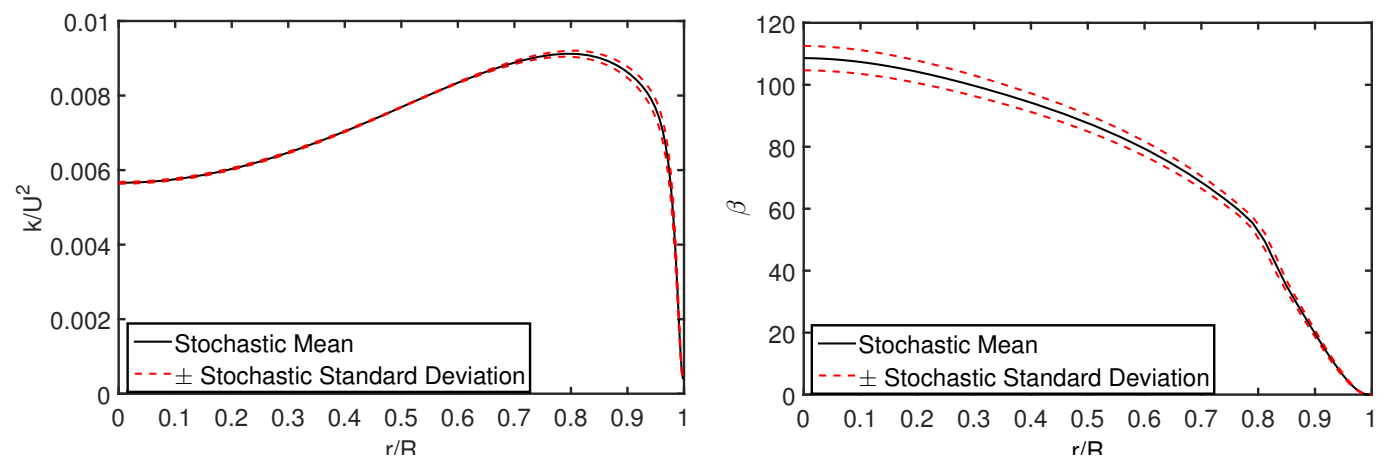

Figure 19: Radial distribution of the dimensionless turbulent kinetic energy and turbulent viscosity ratio profiles at the exit of the pipe for the level 4 of the C-C Sparse Grid at $R e=23000$ and $S=1$.

and variance, for the $N u$ are presented in Tables $5 \& 6$. The radial distribution of the Nusselt number along the plate is shown in Fig. 20, where it is plotted with its standard deviation as uncertainty measure. It can be observed that the most sensitive part to the input uncertainties is the stagnation area, whereas for $x / D$ values far from the stagnation, input uncertainties are irrelevant. In the same manner as in the UQ study in Simulation 1, it can be noted that even by using fewer points for the C-C Sparse Grid, statistical convergence in $N u_{0}$ and $N u_{\text {avg }}$ is generally achieved. Only the stochastic variance needed 29 collocation points to achieve convergence. In addition, since the results are integrated quantities, it is frequent to exhibit some numerical variability due to precision error that do not necessarily mean any oscillation in the convergence rate 
of the UQ technique.

To compare with existing experimental data, most experimentalists studying jets at Reynolds number at/close to our $R e=23000$ test case have reported experimental uncertainty in the Nusselt distribution as a maximum percentage of uncertainty. In our results, the maximum uncertainty is $2.2 \%$, which occurs in the stagnation area. In [14], for $R e=23000$ and $S=0.77$ the reported uncertainty of the Nusselt distribution is below a $3.33 \%$ (this said maximum value corresponds to their largest spacing of $H / D=10$ ). In [84], they estimate a maximum of a $3 \%$ uncertainty for the Nusselt distribution in their swirling impinging jet flows, but they do not specify at which $H / D$, whose value is ranged between 2 and 10. Finally, in [18] an uncertainty estimation of a $2.4 \%$ is reported for their non-swirling impinging jets at $R e=23750$, which is almost identical to the uncertainty in our simulation. We can thence conclude that our computational uncertainty estimation is realistic, according to existing experimental uncertainty on swirling impinging jets from the literature.

\begin{tabular}{cccc}
\hline Level & Points & $N u_{0}$ & $N u_{\text {avg }}$ \\
\hline 1 & 5 & 187.8183333 & 51.45473951 \\
2 & 13 & 187.7914166 & 51.45413825 \\
3 & 29 & 187.8031984 & 51.45457487 \\
4 & 65 & 187.7993255 & 51.45497083 \\
\hline
\end{tabular}

Table 5: Stochastic means of the Nusselt number at the stagnation point and of the surface average over the flat plate.

\begin{tabular}{cccc}
\hline Level & Points & $N u_{0}$ & $N u_{\text {avg }}$ \\
\hline 1 & 5 & 15.24348189 & 1.50945541 \\
2 & 13 & 15.56881637 & 1.50878302 \\
3 & 29 & 15.47284719 & 1.50909036 \\
4 & 65 & 15.47621431 & 1.50822473 \\
\hline
\end{tabular}

Table 6: Stochastic variance of the Nusselt number at the stagnation point and of the surface average over the flat plate.

In order to give a wider insight of the propagation of uncertainties, it is recommended to plot the Probabilistic Distribution Functions (PDF) of the outputs. To obtain such information, Latin Hypercube Sampling (LHS) (see Fig. 21), has been applied on the construction from Eq. (4). With this technique, since the response surface has a negligible cost of evaluation, it has been sampled with 5 million samples to get converged PDFs.

The PDFs for the Nusselt average and at the stagnation point are plotted in Fig. 22. These outputs accurately approximate a $N u_{0} \sim \operatorname{Unif}(181,194.5)$ and $N u_{\text {avg }} \sim \operatorname{Unif}(49.32,53.6)$ distributions. The reasons of having uniform distributions also for the output variables seems to be related to the already discussed predominant linear input-output relationship. The input uncertain parameters have been also modelled as truncated Gaussian distributions to observe their 


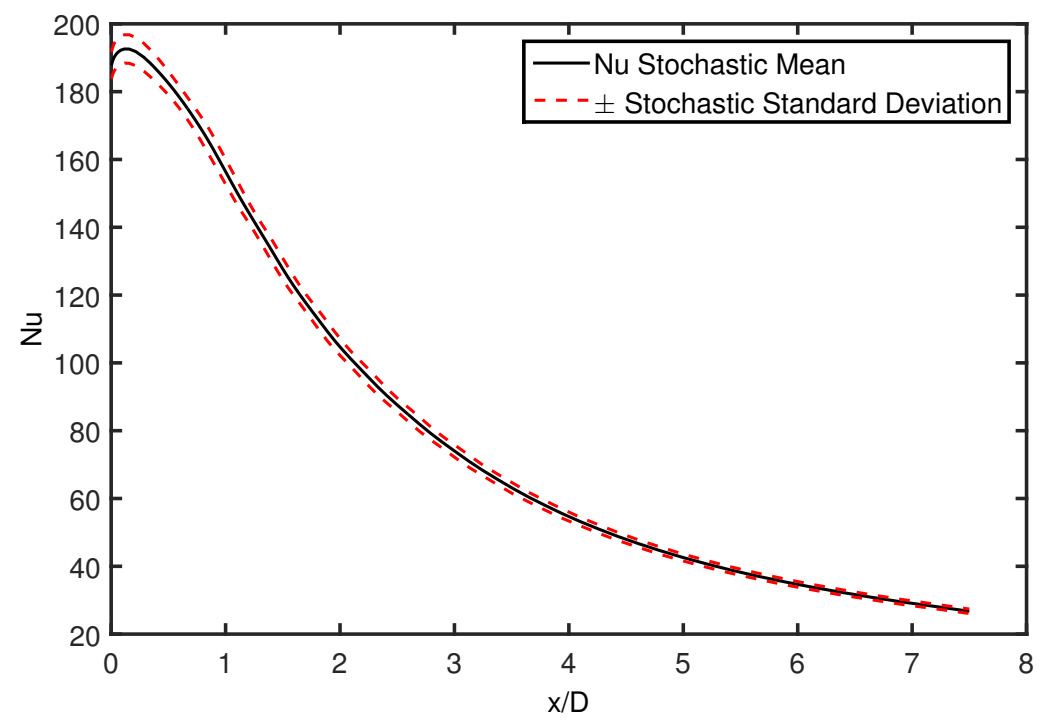

Figure 20: Radial distribution of the stochastic mean of the Nusselt number over the plate \pm its stochastic standard deviation for level 4 of the C-C sparse grid.

contribution to the probabilistic functions of the output. For such constructions, the definition of the input from a standard Gaussian distribution is defined as

$$
\begin{gathered}
Q=\bar{Q}+\sigma_{Q} \theta, \\
\Omega=\bar{\Omega}+\sigma_{\Omega} \theta,
\end{gathered}
$$

with

$$
\begin{aligned}
\theta & \sim N(0,1), \\
\sigma_{Q} & =\operatorname{CoV}_{Q} \bar{Q}, \\
\sigma_{\Omega} & =\operatorname{CoV}_{\Omega} \bar{\Omega},
\end{aligned}
$$

where $N$ refers to the Gaussian distribution, $C o V$ the coefficient of variation $(2.5 \%$ for $Q$ and $0.25 \%$ for $\Omega$ ), $\theta$ the normal distribution of zero value mean and unit variance, and $\sigma$ the standard deviation.

In this paper, the truncated Gaussian distribution is denoted as $\mathcal{N}\left(\mu_{o}, \sigma_{o}^{2} ; \Delta_{l}, \Delta_{u}\right)$, where $\mu_{o}$ and $\sigma_{o}^{2}$ are the mean and variance of the original Gaussian distribution respectively, and $\Delta_{l}$ and $\Delta_{u}$ the lower and upper limits that define the truncation interval.

The coefficient of variation of the original Gaussian distributions have been chosen as half of the corresponding ranges in the considered uniform probabilistic distributions. Since the objective is just to observe the propagation of the uncertainty by different distributions, the coefficients of variation can be set at a lower value if necessary. Also, the shorter the variance, the less the effect of truncation. In our truncated Gaussian functions, the distributions lie within an interval, which is the one defined by the uniform distributions. In other words, these are restricted to the intervals $Q \in(0.95 \bar{Q}, 1.05 \bar{Q})$ and $\Omega \in(0.995 \bar{\Omega}, 1.005 \bar{\Omega})$. An example of their sampling is shown in Fig. 23 for $N_{s}=2000$ samples. 


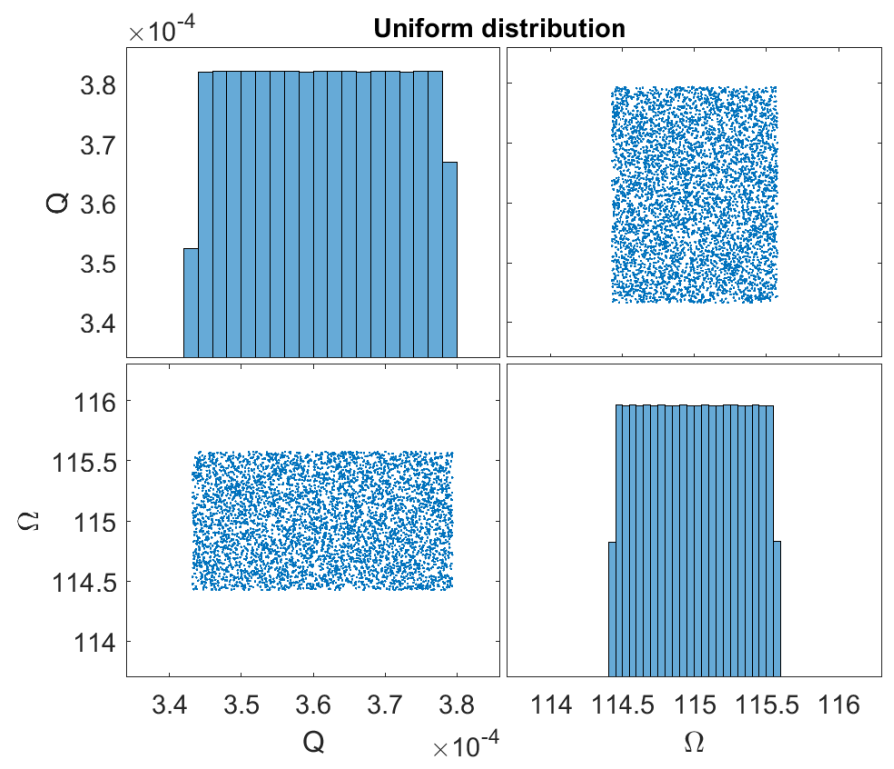

Figure 21: PDFs for $N u_{0}$ (left) and $N u_{\text {avg }}$ (right) when sampling the uniform probabilistic distributions.
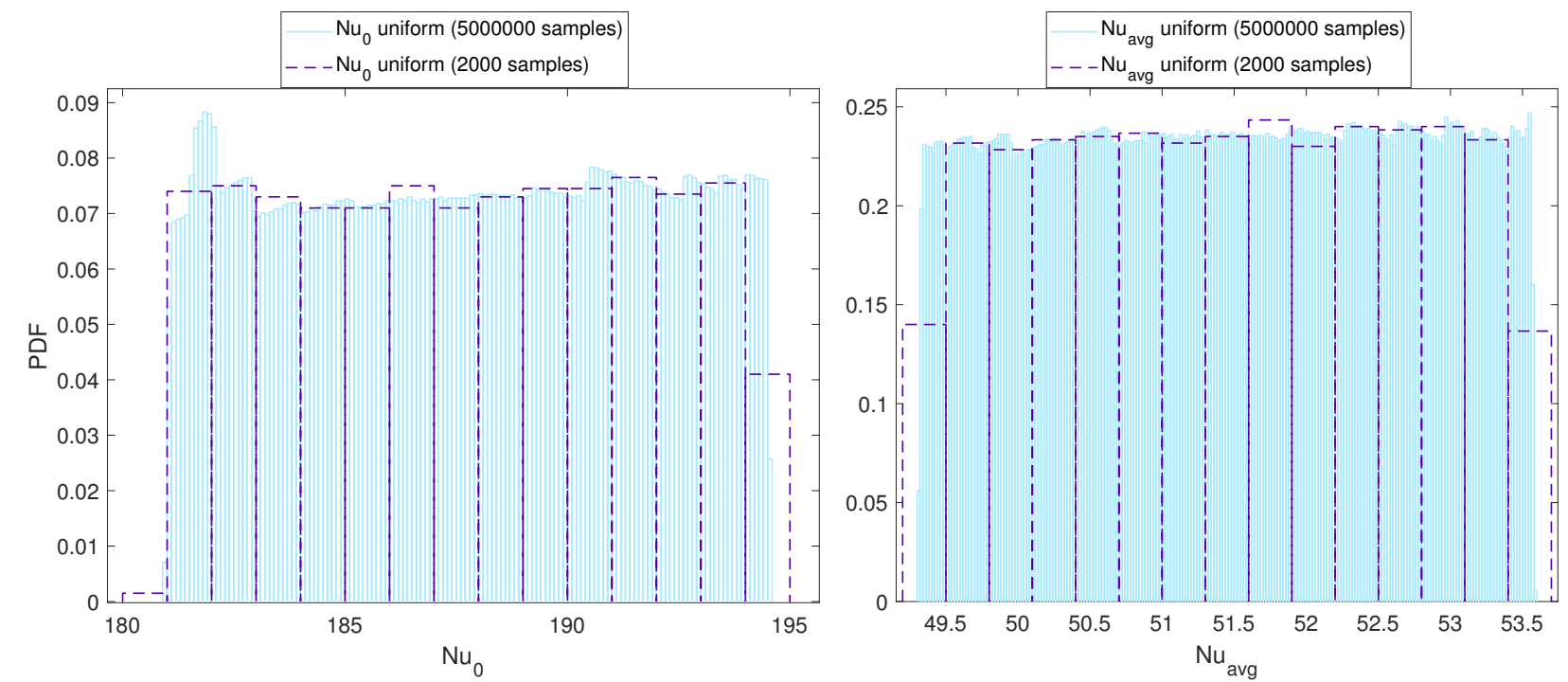

Figure 22: PDFs for $N u_{0}$ (left) and $N u_{\text {avg }}$ (right).

The distributions of the output variables are plotted in Fig. 24 for two different number of samples by means of the LHS method. Again, $N_{s}=5000000$ samples have been used to evaluate the response surface and get a converged probabilistic distribution. The output variables follow the truncated Gaussian distributions $N u_{0} \sim \mathcal{N}(187.9,3.4 ; 181,194.5)$ and $N u_{\text {avg }} \sim$ $\mathcal{N}(51.4,1.06 ; 49.32,53.6)$. The sampling of these estimated distributions is plotted in Fig. 24 in red, with an accurate match. The type of distribution of the inputs has been again preserved, as expected. In addition, one can see in Table 7 and 8 a comparison of the mean and variance of 


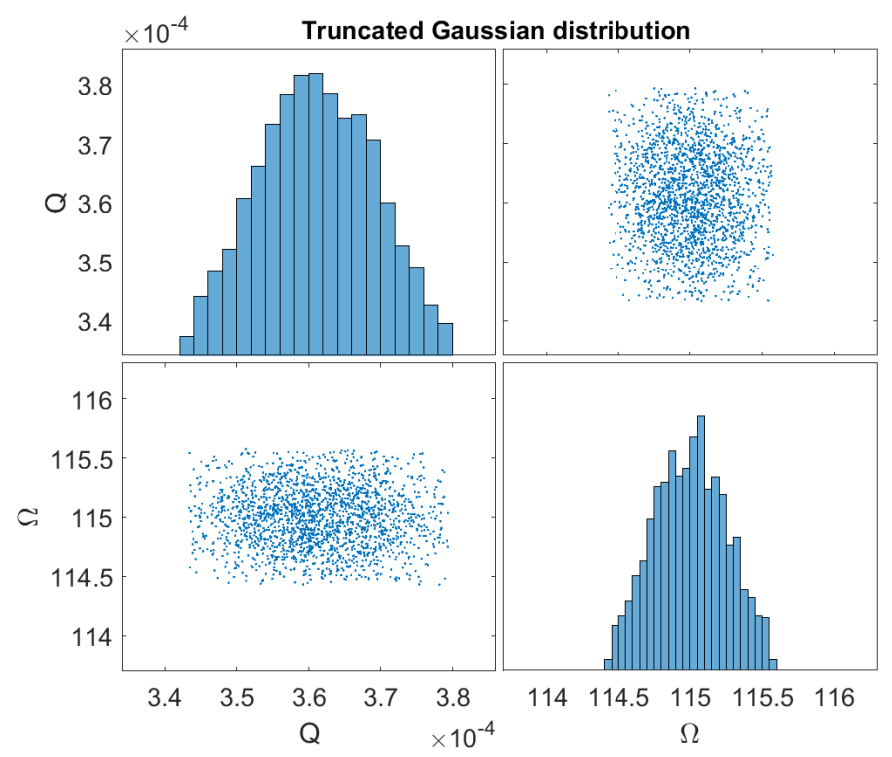

Figure 23: Sampling with $N_{s}=2000$ samples.

the Nusselt number predicted with the uniform and with the Gaussian inputs. It can be noticed in Table 8 that the variances from the Gaussian input are almost half of the corresponding values using a uniform one. This is because the coefficient of variance was halved for the original Gaussian ones and the predominant linearity preserves this proportion.
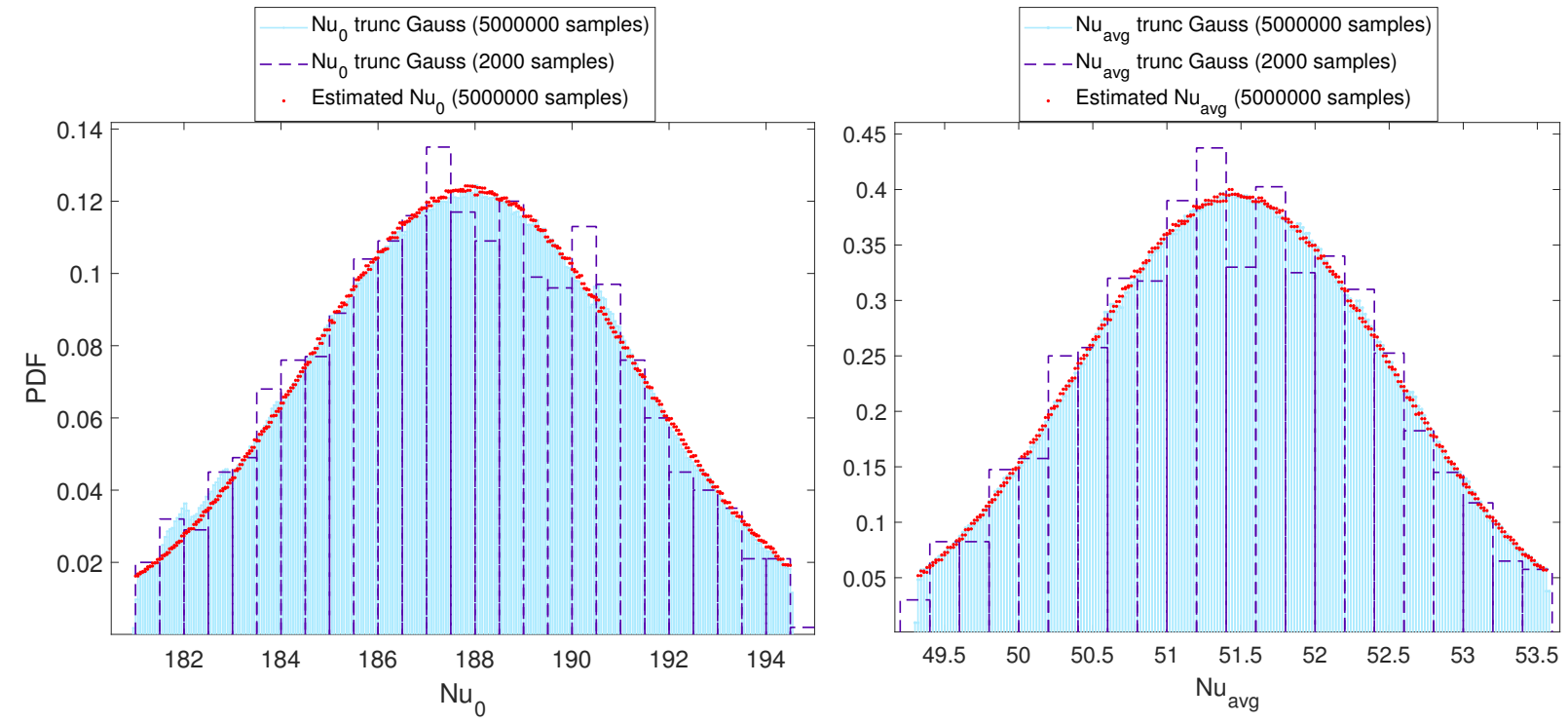

Figure 24: PDFs for $N u_{0}$ (left) and $N u_{\text {avg }}$ (right) when sampling the truncated normal probabilistic distributions. 


\begin{tabular}{ccc}
\hline Input uncertainties & $N u_{0}$ & $N u_{\text {avg }}$ \\
\hline Uniform & 187.80547444 & 51.45682557 \\
Gaussian & 187.82027016 & 51.45935403 \\
\hline
\end{tabular}

Table 7: Stochastic means of the Nusselt number at the stagnation point and its average value along the flat plate for the two different input uncertainties by sampling the response surface from the SCM.

\begin{tabular}{ccc}
\hline Input uncertainties & $N u_{0}$ & $N u_{\text {avg }}$ \\
\hline Uniform & 15.44029670 & 1.50541134 \\
Gaussian & 9.01274706 & 0.87362700 \\
\hline
\end{tabular}

Table 8: Stochastic variance of the Nusselt number at the stagnation point and its average value along the flat plate for the two different input uncertainties by sampling the response surface from the SCM.

\subsection{Simulation 2. Uncertainty Quantification of the Impinging Swirling Jet for Heat Transfer: Use of Non-linear Regression Models for Simulation 1 input.}

In Section 7.2, the computed CFD profiles have been used as new inlet conditions for Simulation 2. However, the use of the modelled regression profiles is another option to run Simulation 2. The outputs by this approach are compared to those by running Simulation 1. Since the collocation points are the same, the difference between these outputs is pointing out the impact of the modelling errors.

To quantify the propagation of uncertainties, the Stochastic Collocation method has been applied for levels 1 and 2 of the Clenshaw-Curtis sparse grid, in order to compare the use of the inlet regression models with the direct CFD inlet condition case (coupling between Simulation 1 and 2). The numerical results are shown in Tables 9 and 10. Note that negative values in the relative error show an increase of the Nusselt number when using the regression models. It can be seen that, despite the models had a very accurate goodness of fit with CFD data profiles in the modelling stage, the small fitting errors are propagated through the simulation leading to a relative error of almost a $8.7 \%$ in the Nusselt number at the stagnation point with respect to the direct CFD inlet condition case. Such percentage of error may seem large in principle, however, by plotting the radial distribution of $N u$ for both the modelled and direct CFD approach (Fig. 25), it can be noticed that the results are not remarkably different. Therefore, the suggested regression models for the inlet of Simulation 2 are introducing very low uncertainty.

\section{Conclusions}

A framework for computational simulations of a two-step heat transfer mechanical device has been carried out to model the propagation of uncertainty, in order to measure the impact of experimen- 


\begin{tabular}{cccccccc}
\hline Level & Points & $N u_{0}$ & $N u_{\text {avg }}$ & $N u_{0, \text { model }}$ & $N u_{\text {avg,model }}$ & $\epsilon_{r, N u_{0}}(\%)$ & $\epsilon_{r, N u_{\text {avg }}}(\%)$ \\
\hline 1 & 5 & 187.81833 & 51.454739 & 188.886500 & 51.245621 & -0.5687 & 0.4064 \\
2 & 13 & 187.79141 & 51.454138 & 188.859827 & 51.244494 & -0.5689 & 0.4074 \\
\hline
\end{tabular}

Table 9: Stochastic means of the Nusselt number case at the stagnation point and its average value along the flat plate. Relative error (in \%) between the CFD and model input results.

\begin{tabular}{cccccccc}
\hline Level & Points & $N u_{0}$ & $N u_{\text {avg }}$ & $N u_{0, \text { model }}$ & $N u_{\text {avg }, \text { model }}$ & $\epsilon_{r, N u_{0}}(\%)$ & $\epsilon_{r, N u_{\text {avg }}}(\%)$ \\
\hline 1 & 5 & 15.243481 & 1.509455 & 16.467074 & 1.521298 & -8.026989 & -0.784601 \\
2 & 13 & 15.568816 & 1.508783 & 16.930460 & 1.521590 & -8.745970 & -0.848853 \\
\hline
\end{tabular}

Table 10: Stochastic variances of the Nusselt number case at the stagnation point and its average value along the flat plate. Relative error (in \%) between the CFD and model input results.

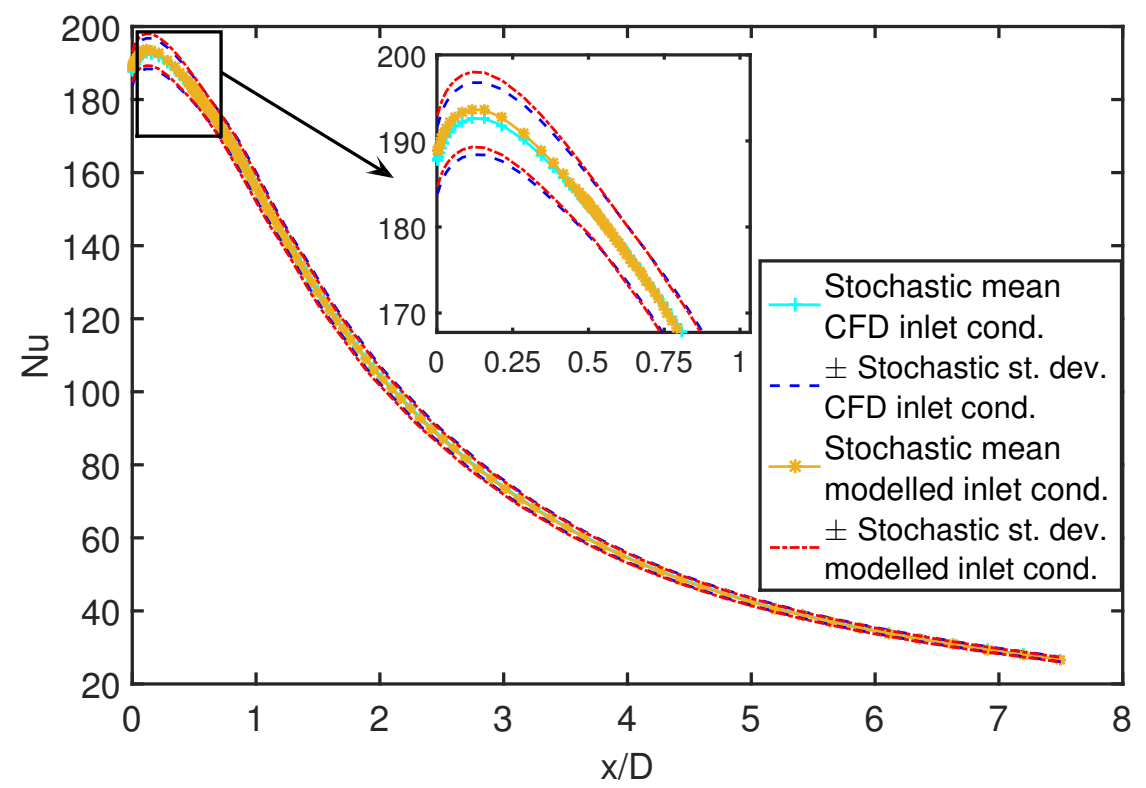

Figure 25: Comparison between radial distribution of the Nusselt number when using the CFD profiles and regression models for the inlet boundary conditions.

tal (aleatoric) uncertainties on the computational performance. The outflow from Simulation 1 (generation of a fully-developed swirling turbulent flow due to the rotation of a round pipe mechanism) has been mathematically modelled, in order to provide numerical stochastic radial profiles for turbulent and velocity variables. The Stochastic Collocation Method with Clenshaw-Curtis nested rule has been applied considering the angular velocity $\Omega$ and the volume flow rate $Q$ as input uncertainties.

In the UQ study on Simulation 1, results have shown that the considered experimental un- 
certainties in $Q$ and $\Omega$ slightly vary the computation of the friction factor, $\lambda$, and the turbulent intensity, $I$. In the dimensionless velocity profiles, these uncertainties have only a modest impact. The most sensitive part of the dimensionless axial velocity profile is near $r / R=0$, and near $r / R=1$ for the azimuthal one. All the axial velocity experimental data lies within the standard deviation envelopes. The azimuthal velocity profiles matched the trend of the experimental data well, but the magnitude is systematically slightly over-predicted by the base case CFD simulation. In the computational uncertainty analysis of the dimensionless turbulent kinetic energy, $k / U^{2}$, the most sensitive region is the part located at the beginning of the decay, due to the strong effect of the wall. Finally, for the laminar to turbulent viscosity ratio, $\beta$, the sensitivity trend is very similar to the dimensionless axial velocity one, exhibiting around the nozzle axis the largest variances. This is the most sensitive computational profile to the input uncertainties, but not remarkable.

The UQ study on the heat transfer process in Simulation 2 was developed in two different ways: by imposing the dimensionless outflow profiles from Simulation 1, and by imposing the non-linear regression models by User Defined Functions as inlet boundary conditions to Simulation 2. With both approaches, it was observed that the random inputs have a modest impact on the predicted Nusselt number along the plate. It was also noticed that $Q$ was the most influential parameter, since $\Omega$ had a very low contribution to uncertainty. As a result, in an experimental facility with similar sources of uncertainty, engineers should put more effort in reducing the stochastic variance in the volume-flow rate by, e.g., reducing pressure losses.

Within the tested ranges of $Q$ and $\Omega$, it has been observed a linear relationship of most quantities of interest analysed, with respect to the uncertain inputs. As a consequence, in most uncertainty results presented in this paper can be noticed that, even by using few collocation points with the Clenshaw-Curtis sparse grid, the statistical moments do not change dramatically. Only the convergence in the stochastic variance of the Nusselt number required a greater number of collocation points (i.e. CFD simulations of Simulation 1 and 2) with important computational efforts.

Four non-linear parametric regression models have been suggested for the fully-developed state of the swirling flow confined in the rotating pipe and used as jet nozzle (dimensionless profiles $v_{z} / U, v_{t} / U, k / U^{2}$ and $\beta$ ). The objective was to provide computational models for one of the most studied impinging jet regimes in the literature with/without swirl: the $R e=23000$. These models fitted well with the computational data from Simulation 1. It was shown that the sensitivity of the distribution of the Nusselt number to the modelling errors is negligible, when the regression

models are imposed onto Simulation 2. Thus, these models are recommended to replace the use of deterministic profiles or stochastic computation of Simulation 1, and can be an interesting option to save computational costs in related engineering applications such as systems with arrays of impinging jets, inlet piping in combustion or chemical mixing, swirling jets impinging on a plate with variations on the surface, or swirling turbulent flows with axisymmetric sudden expansions.

\section{Acknowledgments}

The first author acknowledges his support from the European Community's Seventh Framework Programme (FP7) Marie Curie AeroTraNet 2 Action, under the grant number PITN-GA-2012317142. The second author thanks the III Plan Propio at University of Málaga, which supported 
his visit to the University of Greenwich in Summer 2014. The first author is also thankful to Dr Paul Constantine about the fruitful discussions in the beginning of this work and a warm special acknowledgement to Dr Jeroen A. S. Witteveen for his useful suggestions and who sadly passed away.

\section{References}

[1] W. Song, A. Keane, H. Eres, G. Pound, S. Cox, Two dimensional airfoil optimisation using CFD in a grid computing environment, in: European Conference on Parallel Processing, Springer, 2003, pp. 525-532.

[2] F.-J. Granados-Ortiz, J. Ortega-Casanova, C.-H. Lai, Modelling for computational cost reduction and optimisation of an impinging swirling jet created by a rotating pipe with application to heat transfer from a heated solid flat plate, in: 8th International Congress on Industrial and Applied Mathematics. Beijing, (China). 10-14 August 2015, 2015.

[3] D. McDaniel, R. Cummings, K. Bergeron, S. Morton, J. Dean, Comparisons of CFD solutions of static and maneuvering fighter aircraft with flight test data, Tech. rep., DTIC Document (2007).

[4] F.-J. Granados-Ortiz, C.-H. Lai, C. Perez Arroyo, G. Puigt, C. Airiau, Uncertainty quantification and sensitivity analysis applied to an under-expanded single jet, in: AIAA Aviation 2016, Washington D.C., USA, 2016.

[5] F.-J. Granados-Ortiz, C. P. Arroyo, G. Puigt, C.-H. Lai, C. Airiau, On the influence of uncertainty in computational simulations of a high-speed jet flow from an aircraft exhaust, Computers \& Fluids 180 (2019) 139-158.

[6] S. Taylor, E. Galea, M. Patel, M. Petridis, B. Knight, J. Ewer, SMARTFIRE: an integrated computational fluid dynamics code and expert system for fire field modelling, Fire Safety Science 5 (1997) 1285-1296.

[7] J. Ortega-Casanova, F. Granados-Ortiz, Numerical simulation of the heat transfer from a heated plate with surface variations to an impinging jet, International Journal of Heat and Mass Transfer 76 (2014) 128-143.

[8] J. Mahaffy, B. Chung, C. Song, F. Dubois, E. Graffard, F. Ducros, M. Heitsch, M. Scheuerer, M. Henriksson, E. Komen, et al., Best practice guidelines for the use of CFD in nuclear reactor safety applications, Tech. rep., Organisation for Economic Co-Operation and Development (2007).

[9] F.-J. Granados-Ortiz, J. Ortega-Casanova, C.-H. Lai, Two-step numerical simulation of the heat transfer from a flat plate to a swirling jet flow from a rotating pipe, International Journal of Numerical Methods for Heat and Fluid Flow 30 (1) (2019) 143-175. 
[10] I. B. Celik, U. Ghia, P. J. Roache, et al., Procedure for estimation and reporting of uncertainty due to discretization in CFD applications, Journal of fluids. Engineering-Transactions of the ASME $130(7)$.

[11] J. Ortega-Casanova, Numerical simulation of the heat transfer from a heated solid wall to an impinging swirling jet, INTECH Open Access Publisher, 2011.

[12] J. Ortega-Casanova, CFD and correlations of the heat transfer from a wall at constant temperature to an impinging swirling jet, International Journal of Heat and Mass Transfer 55 (21) (2012) 5836-5845.

[13] M.-Y. Wen, K.-J. Jang, An impingement cooling on a flat surface by using circular jet with longitudinal swirling strips, International Journal of Heat and Mass Transfer 46 (24) (2003) 4657-4667.

[14] D. H. Lee, S. Y. Won, Y. T. Kim, Y. S. Chung, Turbulent heat transfer from a flat surface to a swirling round impinging jet, International Journal of Heat and Mass Transfer 45 (1) (2002) 223-227.

[15] K. Bakirci, K. Bilen, Visualization of heat transfer for impinging swirl flow, Experimental thermal and fluid science 32 (1) (2007) 182-191.

[16] J. Ortega-Casanova, N. Campos, R. Fernandez-Feria, Experimental study on sand bed excavation by impinging swirling jets, Journal of Hydraulic Research 49 (5) (2011) 601-610.

[17] S. Imao, M. Itoh, T. Harada, Turbulent characteristics of the flow in an axially rotating pipe, International journal of heat and fluid flow 17 (5) (1996) 444-451.

[18] J. Baughn, S. Shimizu, Heat transfer measurements from a surface with uniform heat flux and an impinging jet, Journal of Heat Transfer 111 (4) (1989) 1096-1098.

[19] Z. U. Ahmed, Y. M. Al-Abdeli, M. T. Matthews, The effect of inflow conditions on the development of non-swirling versus swirling impinging turbulent jets, Computers \& Fluids 118 (2015) 255-273.

[20] P. Gulati, V. Katti, S. Prabhu, Influence of the shape of the nozzle on local heat transfer distribution between smooth flat surface and impinging air jet, International Journal of Thermal Sciences 48 (3) (2009) 602-617.

[21] N. Zuckerman, N. Lior, Jet impingement heat transfer: physics, correlations, and numerical modeling, Advances in heat transfer 39 (2006) 565-631.

[22] B. Sagot, G. Antonini, A. Christgen, F. Buron, Jet impingement heat transfer on a flat plate at a constant wall temperature, International Journal of Thermal Sciences 47 (12) (2008) 1610-1619.

[23] Z. U. Ahmed, An experimental and numerical study of surface interactions in turbulent swirling jets, Ph.D. thesis, Edith Cowan University (2016). 
[24] P. Grenson, H. Deniau, Large-eddy simulation of an impinging heated jet for a small nozzleto-plate distance and high reynolds number, International Journal of Heat and Fluid Flow 68 (2017) 348-363.

[25] F. Shum-Kivan, F. Duchaine, L. Gicquel, Large-eddy simulation and conjugate heat transfer in a round impinging jet, in: ASME Turbo Expo 2014: Turbine Technical Conference and Exposition, 2014.

[26] N. Uddin, S. O. Neumann, B. Weigand, LES simulations of an impinging jet: On the origin of the second peak in the nusselt number distribution, International Journal of Heat and Mass Transfer 57 (1) (2013) 356-368.

[27] N. Uddin, Turbulence modeling of complex flows in CFD, Ph.D. thesis, University of Stuttgart (2008).

[28] T. Hällqvist, Large eddy simulation of impinging jets with heat transfer, Ph.D. thesis, Royal Institute of Technology (2006).

[29] H. Huang, T. Sun, G. Zhang, D. Li, H. Wei, Evaluation of a developed sst k- $\omega$ turbulence model for the prediction of turbulent slot jet impingement heat transfer, International Journal of Heat and Mass Transfer 139 (2019) 700-712.

[30] H. Huang, T. Sun, G. Zhang, L. Sun, Z. Zong, Modeling and computation of turbulent slot jet impingement heat transfer using rans method with special emphasis on the developed sst turbulence model, International Journal of Heat and Mass Transfer 126 (2018) 589-602.

[31] J. Ortega-Casanova, S. Castillo-Sanchez, On using axisymmetric turbulent impinging jets swirling as Burger's vortex for heat transfer applications. single and multi-objective vortex parameters optimization, Applied Thermal Engineering 121 (2017) 103-114.

[32] Z. U. Ahmed, Y. M. Al-Abdeli, F. G. Guzzomi, Flow field and thermal behaviour in swirling and non-swirling turbulent impinging jets, International Journal of Thermal Sciences 114 (2017) 241-256.

[33] Z. U. Ahmed, Y. M. Al-Abdeli, F. G. Guzzomi, Impingement pressure characteristics of swirling and non-swirling turbulent jets, Experimental Thermal and Fluid Science 68 (2015) $722-732$.

[34] F.-J. Granados-Ortiz, J. Ortega-Casanova, C.-H. Lai, Uncertainty quantification and modelling of CFD simulations of a swirling turbulent jet created by a rotating pipe for application to heat transfer from a heated solid flat plate, in: 1st ECCOMAS Thematic Conference on Uncertainty Quantification in Computational Sciences and Engineering. Crete Island, Greece, 2015.

[35] W. L. Oberkampf, S. M. DeLand, B. M. Rutherford, K. V. Diegert, K. F. Alvin, Error and uncertainty in modeling and simulation, Reliability Engineering \& System Safety 75 (3) (2002) 333-357. 
[36] P. Grossi, Catastrophe modeling: a new approach to managing risk, Vol. 25, Springer Science \& Business Media, 2005.

[37] M. Carnevale, F. Montomoli, A. D'Ammaro, S. Salvadori, F. Martelli, Uncertainty quantification: A stochastic method for heat transfer prediction using LES, Journal of Turbomachinery 135 (5) (2013) 051021.

[38] M. Mendes, S. Ray, J. Pereira, J. Pereira, D. Trimis, Quantification of uncertainty propagation due to input parameters for simple heat transfer problems, International Journal of Thermal Sciences 60 (2012) 94-105.

[39] A. Arefmanesh, M. Mahmoodi, Effects of uncertainties of viscosity models for al2o3-water nanofluid on mixed convection numerical simulations, International journal of Thermal sciences 50 (9) (2011) 1706-1719.

[40] J. Sieres, A. Campo, Uncertainty analysis for the experimental estimation of heat transfer correlations combining the wilson plot method and the monte carlo technique, International Journal of Thermal Sciences 129 (2018) 309-319.

[41] C. Wang, H. G. Matthies, M. Xu, Y. Li, Evidence-theory-based model validation method for heat transfer system with epistemic uncertainty, International Journal of Thermal Sciences 132 (2018) 618-627.

[42] C. Wang, H. G. Matthies, Non-probabilistic interval process model and method for uncertainty analysis of transient heat transfer problem, International Journal of Thermal Sciences 144 (2019) 147-157.

[43] A. Ndiaye, M. Bauerheim, S. Moreau, F. Nicoud, Uncertainty quantification of thermoacoustic instabilities in a swirled stabilized combustor, ASME Paper No. GT2015-44133.

[44] P. M. Congedo, C. Duprat, G. Balarac, C. Corre, Effects of inlet uncertainties on prediction of turbulent flows using RANS and LES simulations, in: 20th AIAA Computational Fluid Dynamics Conference, 2011, p. 3869.

[45] S. Adya, Uncertainty quantification integrated to computational fluid dynamic modeling of synthetic jet actuators, Ph.D. thesis, Missouri University of Science and Technology (2011).

[46] C. Gorle, M. Emory, G. Iaccarino, Epistemic uncertainty quantification of RANS modeling for an underexpanded jet in a supersonic cross flow, CTR Annual Research Briefs, Center for Turbulence Research, Stanford University, Stanford, CA.

[47] A. Mishra, G. Iaccarino, Uncertainty estimation for reynolds-averaged navier-stokes predictions of high-speed aircraft nozzle jets, AIAA Journal 55 (2017) 3999-4004.

[48] G. Iaccarino, A. A. Mishra, S. Ghili, Eigenspace perturbations for uncertainty estimation of single-point turbulence closures, Physical Review Fluids 2 (2) (2017) 024605. 
[49] A. Saltelli, S. Tarantola, F. Campolongo, M. Ratto, Sensitivity Analysis in Practice: A Guide to Assessing Scientific Models, John Wiley \& Sons, Ltd., 2004.

[50] N. Metropolis, S. Ulam, The Monte Carlo method, Journal of the American statistical association 44 (247) (1949) 335-341.

[51] J. C. Helton, F. J. Davis, Latin hypercube sampling and the propagation of uncertainty in analyses of complex systems, Reliability Engineering \& System Safety 81 (1) (2003) 23-69.

[52] H. Faure, C. Lemieux, Generalized Halton sequences in 2008: A comparative study, ACM Transactions on Modeling and Computer Simulation (TOMACS) 19 (4) (2009) 15.

[53] S. Burhenne, O. Tsvetkova, D. Jacob, G. P. Henze, A. Wagner, Uncertainty quantification for combined building performance and cost-benefit analyses, Building and Environment 62 (2013) 143-154.

[54] L. Mathelin, M. Y. Hussaini, A stochastic collocation algorithm for uncertainty analysis, National Aeronautics and Space Administration, Langley Research Center.

[55] S. Smolyak, Quadrature and interpolation formulas for tensor products of certain classes of functions, Soviet Math. Dokl (1963) 240-243.

[56] I. Babuška, F. Nobile, R. Tempone, A stochastic collocation method for elliptic partial differential equations with random input data, SIAM Journal on Numerical Analysis 45 (3) (2007) $1005-1034$.

[57] S. Sankaran, A. L. Marsden, A stochastic collocation method for uncertainty quantification and propagation in cardiovascular simulations, Journal of biomechanical engineering 133 (3) (2011) 031001.

[58] M. Behnia, S. Parneix, P. A. Durbin, Prediction of heat transfer in an axisymmetric turbulent jet impinging on a flat plate, International Journal of Heat and Mass Transfer 41 (12) (1998) $1845-1855$.

[59] X. Yan, N. Saniei, Heat transfer measurements from a flat plate to a swirling impinging jet, International Heat Transfer Conference August 23-28, Kyongiu, Korea 5 (1998) 497-502.

[60] D. Cooper, D. Jackson, B. E. Launder, G. Liao, Impinging jet studies for turbulence model assessment-I. Flow-field experiments, International Journal of Heat and Mass Transfer 36 (10) (1993) 2675-2684.

[61] S. C. Crow, F. Champagne, Orderly structure in jet turbulence, Journal of Fluid Mechanics 48 (3) (1971) 547-591.

[62] C. Yen, N. Messersmith, Application of parabolized stability equations to the prediction of jet instabilities, AIAA journal 36 (8) (1998) 1541-1544. 
[63] P. Balakumar, Prediction of supersonic jet noise, in: 36th AIAA Aerospace Sciences Meeting and Exhibit, 1998, p. 1057.

[64] C. K. Tam, D. E. Burton, Sound generated by instability waves of supersonic flows. Part 2. Axisymmetric jets, Journal of Fluid Mechanics 138 (1984) 273-295.

[65] E. Piot, G. Casalis, F. Muller, C. Bailly, Investigation of the pse approach for subsonic and supersonic hot jets. detailed comparisons with les and linearized euler equations results, International Journal of Aeroacoustics 5 (4) (2006) 361-393.

[66] C. Airiau, T. Ansaldi, Non viscous sensitivity analysis of noise generation mechanism in a low mach number jet.

[67] C. Speziale, B. Younis, S. Berger, Analysis and modelling of turbulent flow in an axially rotating pipe, Journal of Fluid Mechanics 407 (2000) 1-26.

[68] S. Wallin, A. V. Johansson, An explicit algebraic reynolds stress model for incompressible and compressible turbulent flows, Journal of Fluid Mechanics 403 (2000) 89-132.

[69] S. V. Poroseva, S. C. Kassinos, C. A. Langer, W. C. Reynolds, Structure-based turbulence model: Application to a rotating pipe flow, Physics of Fluids 14 (4) (2002) 1523-1532.

[70] Y. Yamada, S. Imao, Swirling flow in an axially rotating pipe, Transactions of JSME 46 (1980) 1662-1670.

[71] G. Loeven, J. Witteveen, H. Bijl, Probabilistic collocation: an efficient non-intrusive approach for arbitrarily distributed parametric uncertainties, in: Proceedings of the 45th AIAA Aerospace Sciences Meeting and Exhibit, AIAA paper, Vol. 317, 2007.

[72] G. Von Winckel, Fast clenshaw-curtis quadrature, Tech. rep., The Mathworks Central File Exchange, Mar. 2008. URL http://www.mathworks.com/matlabcentral/fileexchange/19063sparse-grid-quadrature/content/spquad.m. (2008).

[73] S. A. Smolyak, Quadrature and interpolation formulas for tensor products of certain classes of functions, in: Dokl. Akad. Nauk SSSR, Vol. 4, 1963, pp. 240-243.

[74] O. Brugière, G. Balarac, C. Corre, O. Métais, E. Flores, et al., Numerical prediction of a draft tube flow taking into account uncertain inlet conditions, in: IOP Conference Series: Earth and Environmental Science, Vol. 15, IOP Publishing, 2012, p. 032039.

[75] S. Bailey, M. Hultmark, J. Monty, P. H. Alfredsson, M. Chong, R. Duncan, J. Fransson, N. Hutchins, I. Marusic, B. McKeon, et al., Obtaining accurate mean velocity measurements in high reynolds number turbulent boundary layers using pitot tubes, Journal of Fluid Mechanics 715 (2013) 642-670.

[76] J. A. Witteveen, A. Doostan, T. Chantrasmi, R. Pecnik, G. Iaccarino, Comparison of stochastic collocation methods for uncertainty quantification of the transonic rae 2822 airfoil, in: Proceedings of workshop on quantification of CFD uncertainties, 2009. 
[77] S. W. Chang, Y. J. Jan, S. F. Chang, Heat transfer of impinging jet-array over convex-dimpled surface, International Journal of Heat and Mass Transfer 49 (17) (2006) 3045-3059.

[78] L. F. Geers, M. J. Tummers, K. Hanjalić, Experimental investigation of impinging jet arrays, Experiments in fluids 36 (6) (2004) 946-958.

[79] B. Guo, T. A. G. Langrish, D. F. Fletcher, Simulation of turbulent swirl flow in an axisymmetric sudden expansion, AIAA journal 39 (1) (2001) 96-102.

[80] P. Platteeuw, Application of the probabilistic collocation method to uncertainty in turbulence models, Ph.D. thesis, Delft University (2008).

[81] A. R. Conn, N. I. Gould, P. L. Toint, Trust region methods, Vol. 1, SIAM, 2000.

[82] P. Manneville, Transition to turbulence in wall-bounded flows: Where do we stand?, Mechanical Engineering Reviews 3 (2) (2016) 15-00684.

[83] A. Michalke, On the inviscid instability of the hyperbolic-tangent velocity profile, Deutsche Versuchsanstalt f. Luft-u. Raumfahrt, 1964.

[84] A. Ianiro, G. Cardone, Heat transfer rate and uniformity in multichannel swirling impinging jets, Applied Thermal Engineering 49 (2012) 89-98. 


\section{A Appendix: Models for the fitting coefficients.}

As aforementioned in Section 7.3, the $\gamma_{i}$ parameters are modelled by polynomial regressions. These are introduced in Eqs (23) - (35). The goodness of the fits can be seen in Figs. 26-29.

From these results, the equations to model $\frac{v_{z}}{U}, \frac{v_{t}}{U}, \frac{k}{U^{2}}$ and $\beta$ are only dependent on $Q$ and $\Omega$, and

ready to use for any application. Note the equations are normalised via $Q_{n}=\frac{Q-\bar{Q}}{\sigma_{Q}^{\prime}}$ and $\Omega_{n}=\frac{\Omega-\bar{\Omega}}{\sigma_{\Omega}^{\prime}}$, where overbar denotes mean values and $\sigma^{\prime}$ stands for the standard deviation.

In Table 11 can also be found the goodness indicators of the fit. As the coefficient of determination $\left(\hat{R}^{2}\right)$ is not the best measure for the goodness of a fit, specially in non-linear cases, the Sum of Squares due to Error (SSE), Adjusted- $\hat{R}^{2}$ and Root Mean Squared Error (RMSE) are also given. Over-fitting has been avoided for all the fits by using the minimum order polynomial that reasonably satisfied the goodness.

$$
\begin{aligned}
& a_{z}=-1.048+0.001532 \Omega_{n}-0.01444 Q_{n}-(4.147 e-05) \Omega_{n}^{2}-0.0009388 \Omega_{n} Q_{n} \\
& +0.01183 Q_{n}^{2}-(5.997 e-05) \Omega_{n}^{3}+(3.648 e-05) \Omega_{n}^{2} Q_{n}-0.0005627 \Omega_{n} Q_{n}^{2} \\
& +0.005394 Q_{n}^{3} \\
& b_{z}=3.023+0.004779 \Omega_{n}-0.05368 Q_{n}+(5.742 e-06) \Omega_{n}^{2}-0.0005031 \Omega_{n} Q_{n} \\
& +0.003906 Q_{n}^{2}, \\
& c_{z}=-0.6536-0.002857 \Omega_{n}+0.03038 Q_{n}+(1.346 e-05) \Omega_{n}^{2}+0.0003585 \Omega_{n} Q_{n} \\
& -0.004234 Q_{n}^{2} \\
& d_{z}=34.16+-0.05319 \Omega_{n}+1.362 Q_{n}+0.0008092 \Omega_{n}^{2}+0.02154 \Omega_{n} Q_{n}-0.3225 Q_{n}^{2} \\
& +0.002316 \Omega_{n}^{3}+0.0001007 \Omega_{n}^{2} Q_{n}+0.01276 \Omega_{n} Q_{n}^{2}-0.1631 Q_{n}^{3}, \\
& a_{t}=0.9708+0.003415 \Omega_{n}-0.03677 Q_{n}-0.0001444 \Omega_{n} Q_{n}+0.001342 Q_{n}^{2}, \\
& b_{t}=2.005-0.006083 \Omega_{n}+0.06979 Q_{n}+0.0004104 \Omega_{n} Q_{n}-0.004283 Q_{n}^{2} \\
& a_{k}=-0.1709-0.0001532 \Omega_{n}-0.000986 Q_{n}+(7.86 e-05) \Omega_{n} Q_{n}+0.0007671 Q_{n}^{2} \\
& -0.0001781 \Omega_{n} Q_{n}^{2}-(6.527 e-05) Q_{n}^{3}-(3.653 e-05) \Omega_{n} Q_{n}^{3}-0.0003428 Q_{n}^{4} \\
& +(8.297 e-05) \Omega_{n} Q_{n}^{4}+0.0001397 Q_{n}^{5}, \\
& b_{k}=-1.698+0.001364 \Omega_{n}-0.002698 Q_{n}+(3.023 e-05) \Omega_{n}^{2}-0.0002644 \Omega_{n} Q_{n} \\
& -0.002849 Q_{n}^{2}+(3.223 e-05) \Omega_{n}^{2} Q_{n}+0.0001315 \Omega_{n} Q_{n}^{2}-0.001438 Q_{n}^{3} \\
& -(6.665 e-05) \Omega_{n}^{2} Q_{n}^{2}+(7.724 e-05) \Omega_{n} Q_{n}^{3}+0.001602 Q_{n}^{4},
\end{aligned}
$$




$$
\begin{gathered}
c_{k}=-0.08801+(5.075 e-05) \Omega_{n}+0.0005716 Q_{n}+(3.703 e-06) \Omega_{n}^{2} \\
-(2.574 e-05) \Omega_{n} Q_{n}+0.0001155 Q_{n}^{2}, \\
a_{\beta}=1.932-0.01402 \Omega_{n}+0.4053 Q_{n}-0.0006922 \Omega_{n} Q_{n}+0.02551 Q_{n}^{2}, \\
b_{\beta}=0.7933+0.001219 \Omega_{n}+0.003093 Q_{n}-(7.063 e-06) \Omega_{n} Q_{n}+0.001437 Q_{n}^{2} \\
+(1.968 e-06) \Omega_{n} Q_{n}^{2}-0.0002586 Q_{n}^{3}, \\
c_{\beta}=1.862-0.000533 \Omega_{n}-0.04129 Q_{n}-0.0002055 \Omega_{n} Q_{n}+0.005018 Q_{n}^{2}, \\
d_{\beta}=4.047+0.008784 \Omega_{n}-0.1622 Q_{n}-0.0009475 \Omega_{n} Q_{n}+0.00729 Q_{n}^{2} .
\end{gathered}
$$
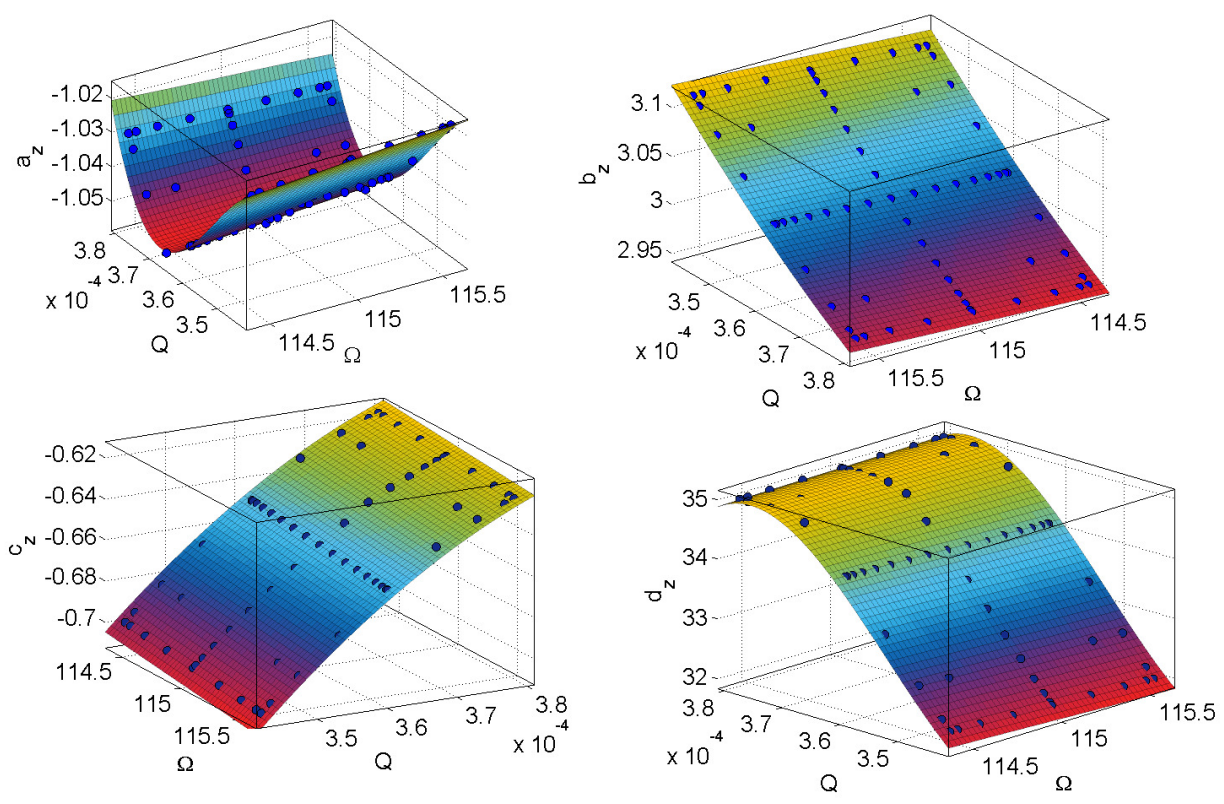

Figure 26: Models for the $\frac{v_{z}}{U}$ coefficients. 

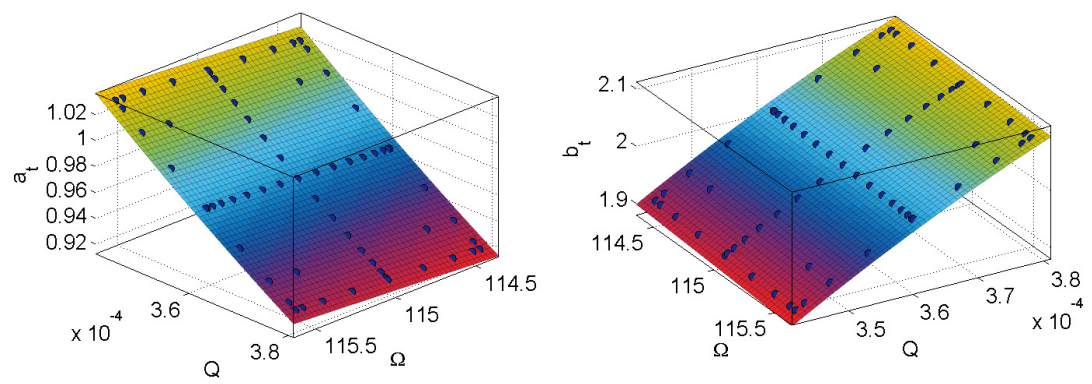

Figure 27: Models for the $\frac{v_{t}}{U}$ coefficients.

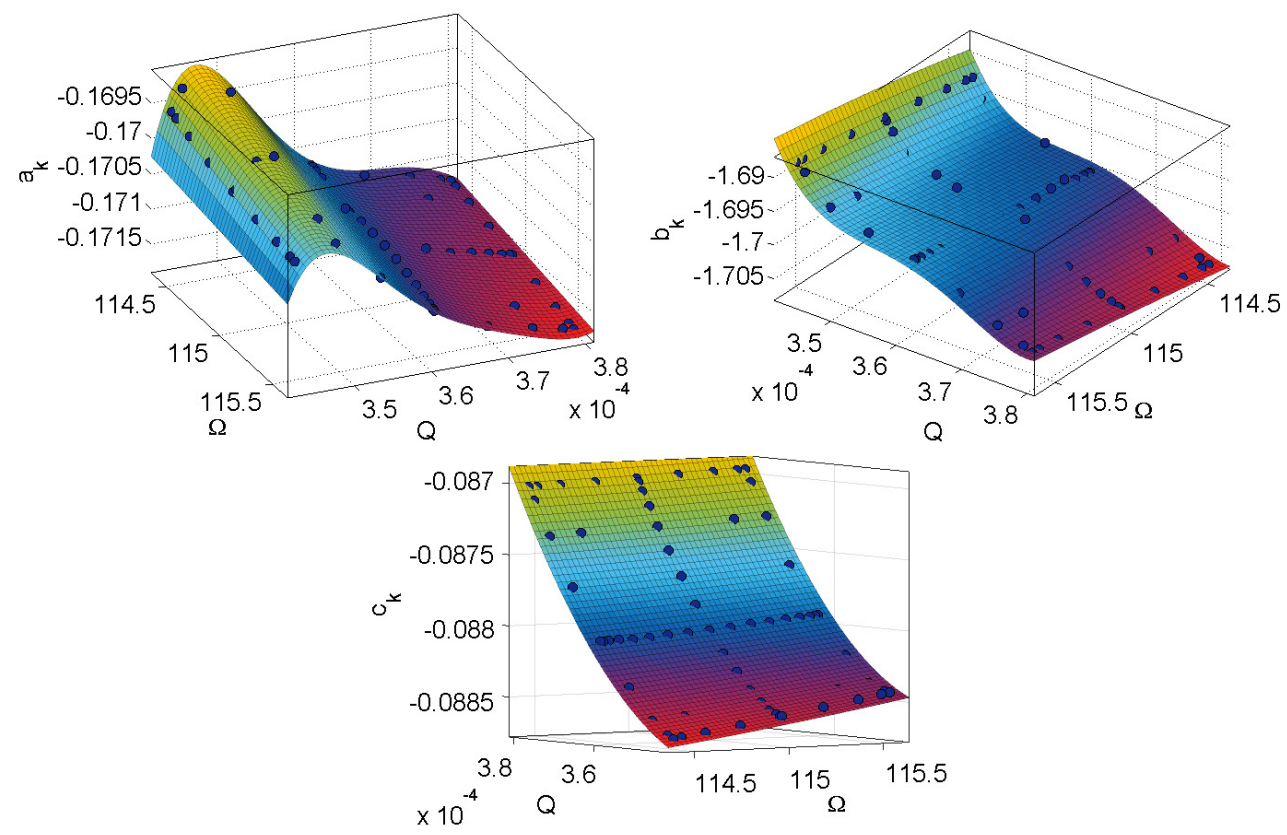

Figure 28: Models for the $\frac{k}{U^{2}}$ coefficients. 

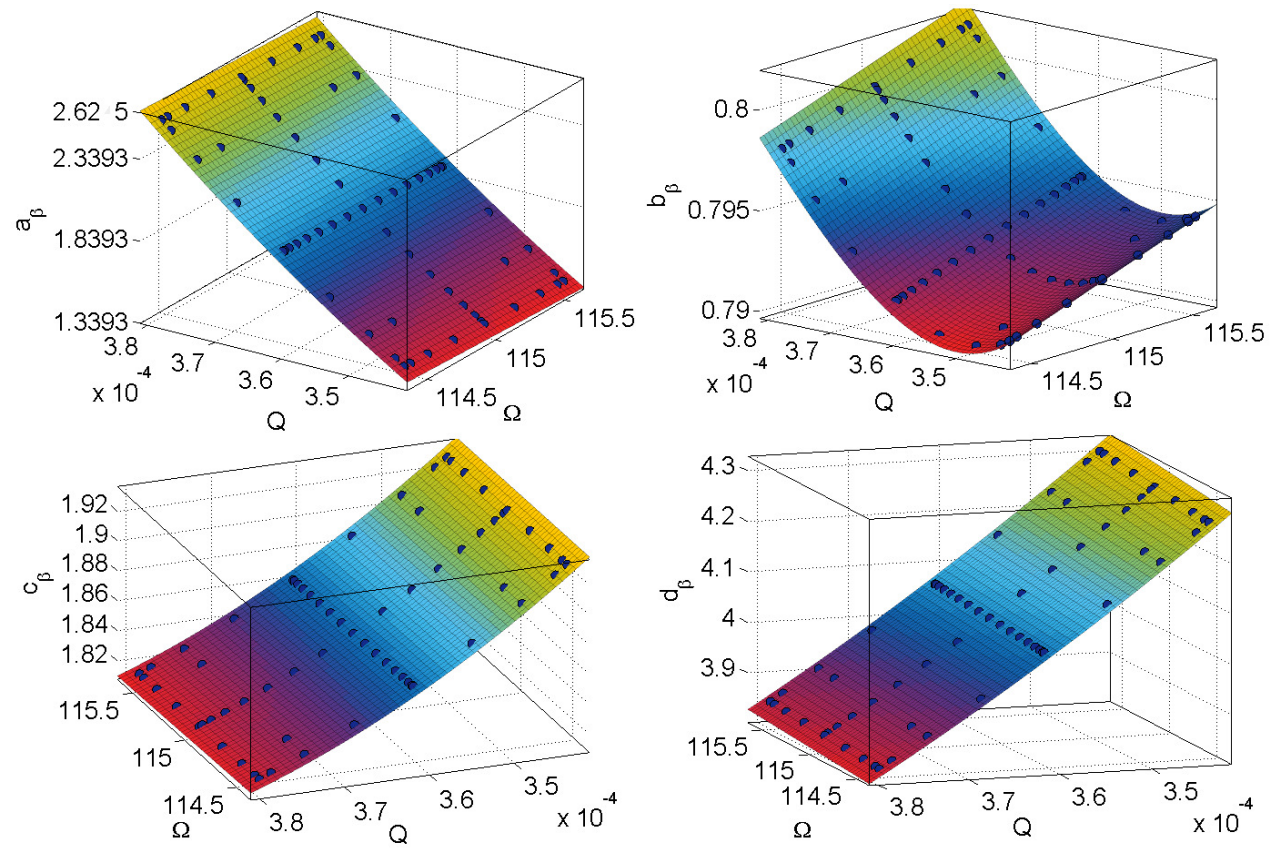

Figure 29: Models for the $\beta$ coefficients.

\begin{tabular}{c|cccc}
\hline Coefficient & SSE & $\hat{R}^{2}$ & Adjusted $\hat{R}^{2}$ & RMSE \\
\hline$a_{z}$ & $8.206 \mathrm{e}-05$ & 0.9903 & 0.9887 & 0.001221 \\
$b_{z}$ & $4.892 \mathrm{e}-07$ & 1 & 1 & $9.105 \mathrm{e}-05$ \\
$c_{z}$ & $3.278 \mathrm{e}-05$ & 0.9995 & 0.9994 & 0.0007453 \\
$d_{z}$ & 0.07246 & 0.9991 & 0.999 & 0.0363 \\
\hline$a_{t}$ & $6.173 \mathrm{e}-08$ & 1 & 1 & $3.208 \mathrm{e}-05$ \\
$b_{t}$ & $4.583 \mathrm{e}-07$ & 1 & 1 & $8.74 \mathrm{e}-05$ \\
\hline$a_{k}$ & $3.357 \mathrm{e}-07$ & 0.9904 & 0.9887 & $7.884 \mathrm{e}-05$ \\
$b_{k}$ & $1.371 \mathrm{e}-05$ & 0.9925 & 0.991 & 0.0005086 \\
$c_{k}$ & $1.238 \mathrm{e}-08$ & 0.9994 & 0.9994 & $1.448 \mathrm{e}-05$ \\
\hline$a_{\beta}$ & $4.878 \mathrm{e}-06$ & 1 & 1 & 0.0002851 \\
$b_{\beta}$ & $2.248 \mathrm{e}-08$ & 1 & 1 & $1.969 \mathrm{e}-05$ \\
$c_{\beta}$ & $1.954 \mathrm{e}-06$ & 1 & 1 & 0.0001805 \\
$d_{\beta}$ & $7.532 \mathrm{e}-06$ & 1 & 1 & 0.0003543 \\
\hline
\end{tabular}

Table 11: Goodness of the fitting for the coefficients of the models. 


\section{B Appendix: User-Defined Functions to implement the models.}

The models have been implemented in FLUENT by means of a User-Defined Function (UDF) coded in $C$. In this appendix, a simple example about how to implement the UDF is given. Please note that the mathematical expressions of the coefficients, introduced in Appendix A should be implemented in the UDF as well. For sake of simplicity, these models are not implemented and the coefficients are supposed to be calculated and used as defined variables in the UDF.

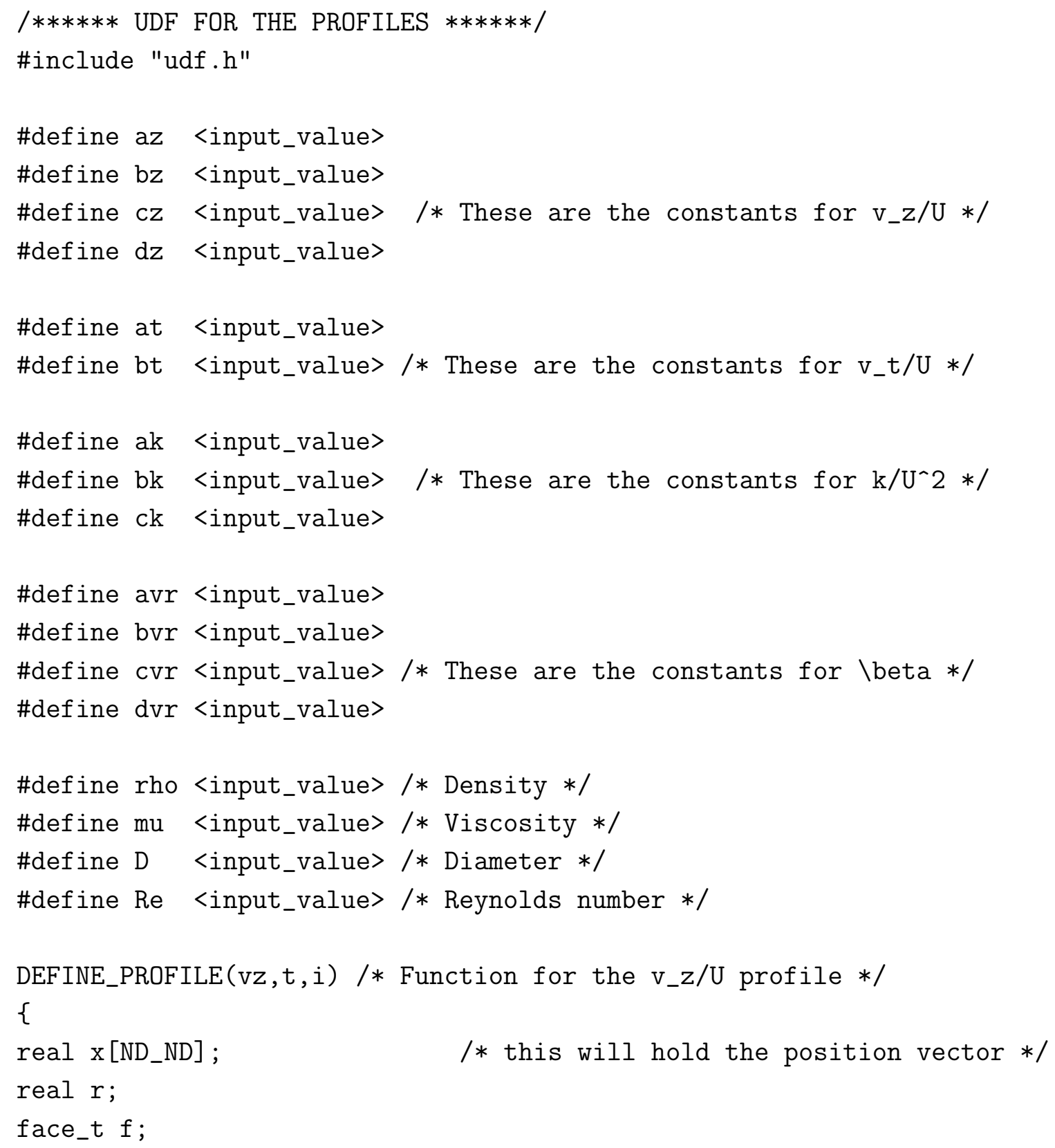




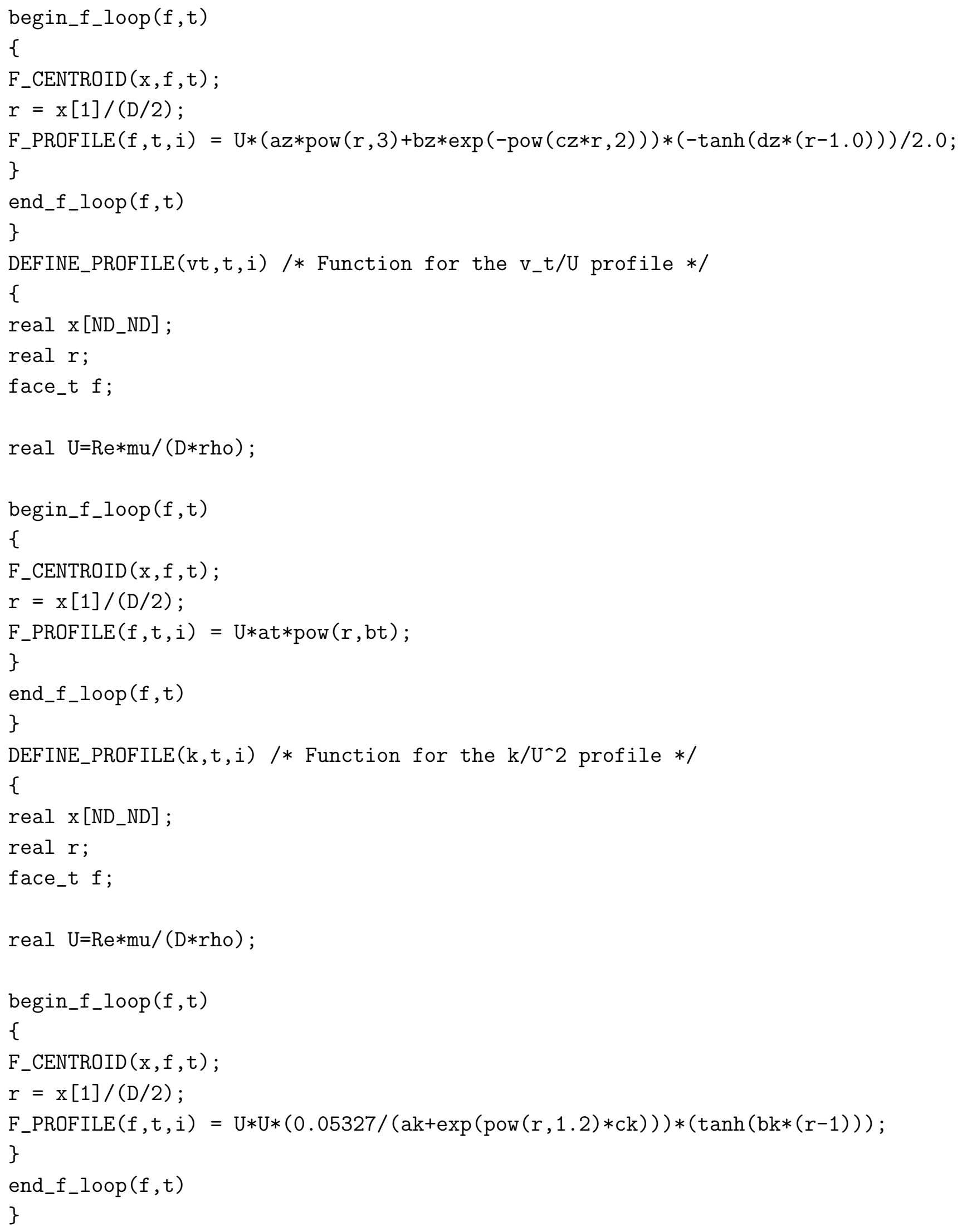




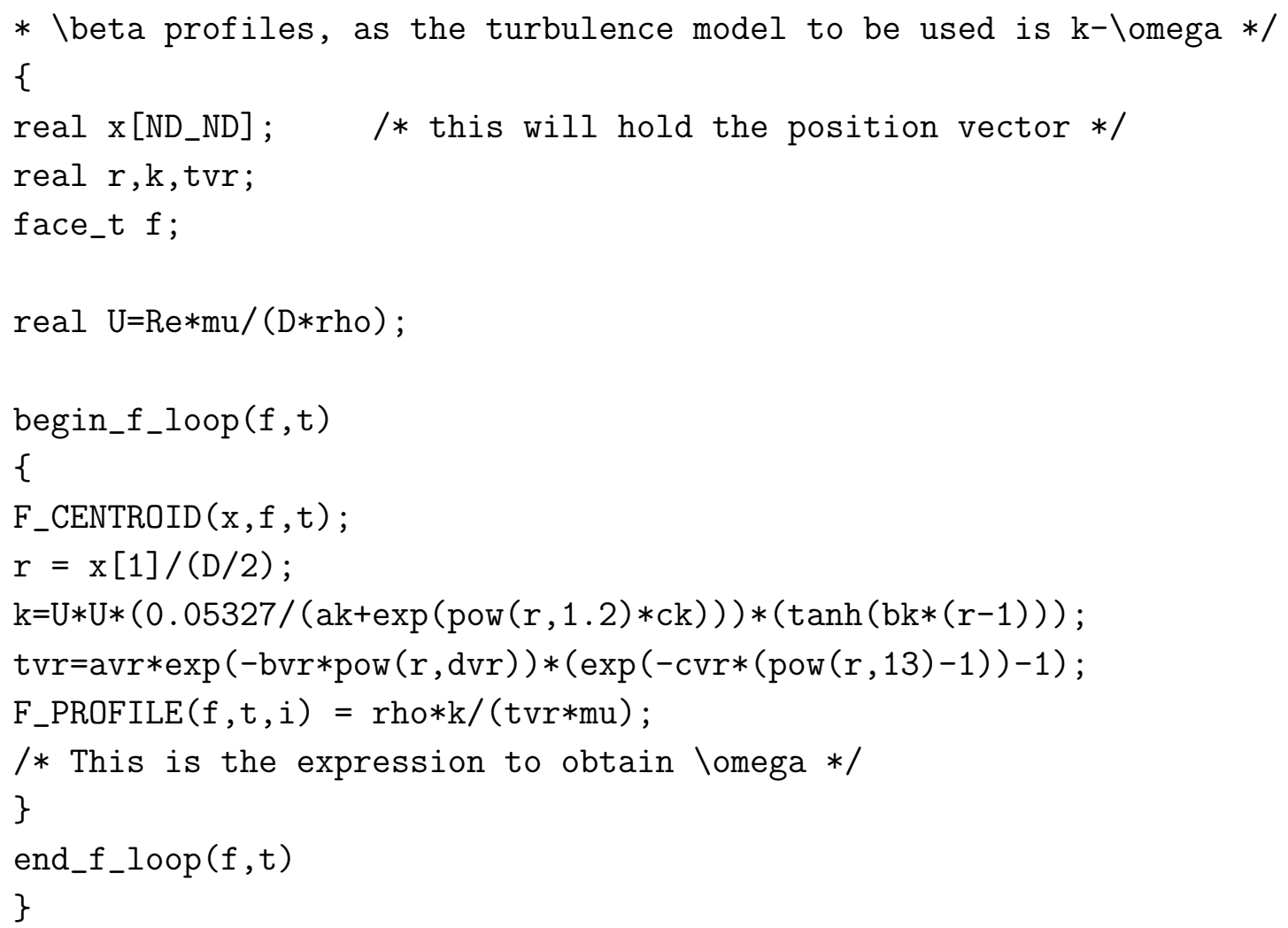

